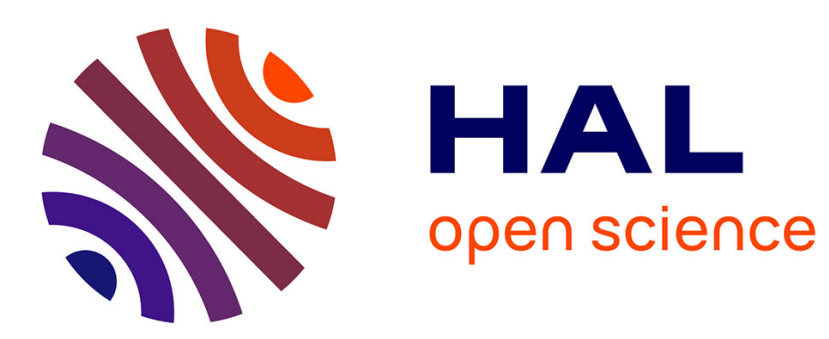

\title{
Theoretical and numerical investigation of the finite cell method
}

\author{
Monique Dauge, Alexander Düster, Ernst Rank
}

\section{To cite this version:}

Monique Dauge, Alexander Düster, Ernst Rank. Theoretical and numerical investigation of the finite cell method. 2013. hal-00850602v1

\section{HAL Id: hal-00850602 \\ https://hal.science/hal-00850602v1}

Preprint submitted on 7 Aug 2013 (v1), last revised 23 Dec 2014 (v3)

HAL is a multi-disciplinary open access archive for the deposit and dissemination of scientific research documents, whether they are published or not. The documents may come from teaching and research institutions in France or abroad, or from public or private research centers.
L'archive ouverte pluridisciplinaire HAL, est destinée au dépôt et à la diffusion de documents scientifiques de niveau recherche, publiés ou non, émanant des établissements d'enseignement et de recherche français ou étrangers, des laboratoires publics ou privés. 


\title{
Theoretical and numerical investigation of the finite cell method
}

\author{
Monique Dauge ${ }^{1}$, Alexander Düster ${ }^{2}$, and Ernst Rank ${ }^{3}$ \\ ${ }^{1}$ Université de Rennes 1, France \\ ${ }^{2}$ Technische Universität Hamburg-Harburg, Germany \\ ${ }^{3}$ Technische Universität München, Germany
}

June 23, 2013

\begin{abstract}
We present a detailed analysis of the convergence properties of the finite cell method which is a fictitious domain approach based on high order finite elements. It is proved that exponential type of convergence can be obtained by the finite cell method for Laplace and Lamé problems in one, two as well three dimensions. Several numerical examples in one and two dimensions including a wellknown benchmark problem from linear elasticity confirm the results of the mathematical analysis of the finite cell method.
\end{abstract}

\section{Introduction}

The finite cell method (FCM) [13, 7, 8] is a combination of a fictitious domain approach $[19,20]$ with finite elements of high order [25, 4]. The main idea is to embed the domain of the problem to be solved into a bigger domain that has a simple geometric shape and can therefore be readily meshed. Thanks to the simple shape of the embedding or fictitious domain, mesh generation is dramatically simplified. The geometry of the problem is considered during the integration of the cell matrices, i.e. when computing the stiffness and mass matrices. To this end an indicator function $\alpha$ is introduced which is equal to 1 inside the domain and 0 outside of the domain. In order to avoid conditioning problems, $\alpha$ is set to a very small value close to zero outside the domain. In this way the contribution of the fictitious domain is penalized, shifting the effort of meshing towards the numerical integration of the cell matrices. Since the quality of the finite cell approximation strongly depends on the accuracy of the numerical integration, an adaptive quadrature scheme is applied to compute the stiffness and mass matrices of cells that are cut by the boundary of the domain or include holes. The adaptive integration can be carried out very generally by applying quadtree (in 2D) and octree (in 3D) space partitioning schemes in a fully automatic, error-controlled fashion [2]. Summarizing, the finite cell method is based on three important ingredients: a fictitious domain approach, high order shape functions and an adaptive integration of the cell matrices. Combining these ingredients allows to achieve an exponential type of convergence when performing a $p$-extension of the trial and test functions of the cells.

The FCM has been applied to several problems like linear elasticity in 2D [13] and 3D [7], to shell problems [16] as well as to problems in biomechanics [5, 26, 27]. Nonlinear problems such 
as geometrically nonlinearity [23] or elastoplasticity [1,3] have been addressed as well. The FCM has also been successfully applied to the numerical homogenization of materials with complicated microstructures [9] or to topology optimization [6, 14] in structural mechanics. Instead of classical hierarchic shape functions [25] NURBS, which have become very popular thanks to the isogeometric analysis [10], can also be successfully used within the FCM, see [22, 17]. Local refinement strategies have been also developed for the FCM and it turned out that the $h p$ - $d$ method $[15,5]$ presents a general framework for local improvement of accuracy within the FCM, see [21, 11].

Despite the fact that the FCM has been numerically demonstrated to yield high convergence rates in many different problems, there is still a lack of a thoroughly mathematical analysis of its convergence properties. Therefore, this paper is devoted to the analysis of the FCM, proving its capabilities of achieving exponential convergence under conditions, which are similar to those for the $p$-version of the finite element method.

The layout of the paper is as follows: In Section 2 the setting of the problem is defined and the convergence of the discrete and continuous problem with respect to the penalization parameter is presented. In Section 3 Céa and Strang lemmas are revisited to check that strict positiveness of the bilinear forms is not necessary. In Section 4 the convergence of the $p$-version of finite elements is addressed which is one of the main ingredients of the FCM. Numerical examples for 1D problems are presented in Section 5 and the observed exponential convergence is proved. In Section 6 a two-dimensional benchmark of linear elasticity is studied and it is demonstrated that the exponential convergence can be also obtained in 2D. Finally, a conclusion is drawn in Section 7.

\section{Neumann condition obtained by penalization}

\subsection{The setting}

We consider a bounded domain $\Omega \subset \mathbb{R}^{n}, n=1,2$ or 3 with reasonable smoothness assumption (regular or polyhedral or Lipschitz...). We assume that the boundary of $\Omega$ has at least two connected components, so that $\Omega$ has a finite number of holes: The complement domain $\Omega^{\prime}:=\mathbb{R}^{n} \backslash \bar{\Omega}$ has one unbounded component $\Omega_{0}^{\prime}$ and a finite number of bounded connected components $\Omega_{j}^{\prime}, j=1, \ldots, J$. We denote by $\mathcal{H}$ the hole

$$
\mathcal{H}=\cup_{j=1}^{J} \Omega_{j}^{\prime},
$$

by $\Gamma$ be the boundary of $\Omega_{0}^{\prime}$, by $\Sigma$ the boundary of $\mathcal{H}$ and by $\mathcal{D}$ the domain with holes removed

$$
\mathcal{D}=\Omega \cup \overline{\mathcal{H}} \text {. }
$$

Note that the boundary of $\mathcal{D}$ is $\Gamma$, the boundary of $\Omega$ is $\Sigma \cup \Gamma$.

The aim is to solve an elliptic equation $L u=f$ on $\Omega$ with Dirichlet conditions on $\Gamma$ and Neumann conditions on $\Sigma$ by solving Dirichlet problems on $\mathcal{D}$. So $\mathcal{D}$ plays the role of a fictitious domain.

More specifically, we are given two differential bilinear forms $b_{0}$ and $b_{1}$ of degree 1 which we assume for simplicity to be real symmetric with constant coefficients (at this point we may consider systems Lamé - as well), e.g.

$$
b_{0}(u, v)(x)=\nabla u(x) \cdot \nabla v(x)+k^{2} u(x) v(x) \quad \text { and } \quad b_{1}(u, v)(x)=\nabla u(x) \cdot \nabla v(x) .
$$


Let us define variational spaces:

$$
V(\Omega)=\left\{u \in H^{1}(\Omega): u=0 \text { on } \Gamma\right\} \quad \text { and } \quad V(\mathcal{D})=H_{0}^{1}(\mathcal{D}) .
$$

and the variational forms

$$
a_{0}(u, v)=\int_{\Omega} b_{0}(u, v)(x) d x \quad \text { and } \quad a_{1}(u, v)=\int_{\mathcal{H}} b_{1}(u, v)(x) d x .
$$

Let $f \in L^{2}(\Omega)$. We want to solve the variational problem

$$
\text { Find } u \in V(\Omega), \quad \forall v \in V(\Omega), \quad a_{0}(u, v)=\int_{\Omega} f(x) v(x) d x .
$$

Instead, we solve for small $\alpha>0$ the following variational problem on $\mathcal{D}$

$$
\text { Find } u_{\alpha} \in V(\mathcal{D}), \quad \forall v \in V(\mathcal{D}), \quad a_{0}\left(u_{\alpha}, v\right)+\alpha a_{1}\left(u_{\alpha}, v\right)=\int_{\mathcal{D}} f(x) v(x) d x \text {. }
$$

Note that

$$
a_{0}(u, v)+\alpha a_{1}(u, v)=\int_{\Omega} b_{0}(u, v)(x) d x+\alpha \int_{\mathcal{H}} b_{1}(u, v)(x) d x .
$$

At the discrete level, we replace a FEM discretization of problem (2.5) by a discretization of (2.6): With a finite dimensional approximation $V^{\mathrm{ap}}(\mathcal{D})$ of $V(\mathcal{D})$, and a numerical integration $\int_{\mathcal{D} \mid \text { ap }}$ over $\mathcal{D}$, the discrete problem with parameter $\alpha$ is

$$
\text { Find } u_{\alpha}^{\mathrm{ap}} \in V^{\mathrm{ap}}(\mathcal{D}), \quad \forall v \in V^{\mathrm{ap}}(\mathcal{D}), \quad a_{0}^{\mathrm{ap}}\left(u_{\alpha}^{\mathrm{ap}}, v\right)+\alpha a_{1}^{\mathrm{ap}}\left(u_{\alpha}^{\mathrm{ap}}, v\right)=\int_{\mathcal{D} \mid \mathrm{ap}} f(x) v(x) d x,
$$

where

$$
a_{0}^{\mathrm{ap}}(u, v)=\int_{\mathcal{D} \mid \mathrm{ap}} \mathbf{1}_{\Omega}(x) b_{0}(u, v)(x) d x \quad \text { and } \quad a_{1}^{\mathrm{ap}}(u, v)=\int_{\mathcal{D} \mid \mathrm{ap}} \mathbf{1}_{\mathcal{H}}(x) b_{1}(u, v)(x) d x .
$$

\subsection{Convergence of discrete problems with respect to the penalization parameter}

Let us consider the finite dimensional space $V=V^{\mathrm{ap}}(\mathcal{D})$ and the numerical integration $\int_{\mathcal{D} \mid \text { ap }}$ as fixed, and let $\alpha$ tend to 0 . We prove in Lemma 1 that, under a simple assumption, problem (2.7) converges to a limit as $\alpha \rightarrow 0$. We introduce the kernel of $a_{0}^{\text {ap }}$

$$
K_{0}=\left\{v \in V: \forall u \in V, a_{0}^{\mathrm{ap}}(u, v)=0\right\},
$$

and its orthogonal space

$$
K_{0}^{\perp}=\left\{\varphi \in V^{\prime}: \forall v \in K_{0},\langle\varphi, v\rangle=0\right\} .
$$

Here $\langle\varphi, v\rangle$ denotes the duality pairing between $V^{\prime}$ and $V$.

We define the operators $\bar{A}_{k}$ for $k=0,1$ :

$$
\begin{aligned}
& \bar{A}_{k}: V \quad \longrightarrow \quad V^{\prime} \\
& u \longmapsto\left(V \ni v \mapsto a_{k}^{\mathrm{ap}}(u, v)\right),
\end{aligned}
$$


And introduce their restrictions

$$
\begin{aligned}
A_{0}: \quad V & \longrightarrow \\
u & \longmapsto \\
& K_{0}^{\perp}
\end{aligned}\left(V \ni v \mapsto a_{0}^{\mathrm{ap}}(u, v)\right),
$$

and

$$
\begin{aligned}
A_{1}: V & \longrightarrow \\
u & \longmapsto\left(K_{0} \ni v \mapsto a_{1}^{\mathrm{ap}}(u, v)\right),
\end{aligned}
$$

and, finally, the operator $A$

$$
\begin{aligned}
A: V & \longrightarrow K_{0}^{\perp} \times K_{0}^{\prime} \\
u & \longmapsto
\end{aligned}
$$

Lemma 1 In the finite dimensional framework, if $\left.A_{1}\right|_{K_{0}}$ is bijective, then $A$ is bijective. Let $f \in K_{0}^{\perp}$. Then for $\alpha$ small enough, problem (2.7) has a unique solution $u_{\alpha}$. Let $u_{0}$ be defined as $A^{-1}(f, 0)$. Then $u_{\alpha}$ tends to $u_{0}$ in $V$, and in any fixed norm $\|\cdot\|$ on $V$

$$
\left\|u_{\alpha}-u_{0}\right\| \leq C_{A} \alpha\|f\| .
$$

Proof: $\quad A_{0}$ sends $V$ into $K_{0}^{\perp}$, and $A$ sends $V$ into $K_{0}^{\perp} \times K_{0}^{\prime}$. These two latter spaces have the same dimension. Since $\left.A_{1}\right|_{K_{0}}$ is bijective, the kernel of $A$ is reduced to $\{0\}$, hence the bijectivity of $A$.

We define recursively:

$$
u^{(0)}:=u_{0}=A^{-1}(f, 0) \quad \text { and } \quad u^{(j)}=-A^{-1}\left(\bar{A}_{1} u^{(j-1)}, 0\right), j=1,2, \ldots
$$

This makes sense, since by definition $A_{1} u^{(j-1)}=0$, hence $\bar{A}_{1} u^{(j-1)}$ belongs to $K_{0}^{\perp}$.

Then, for $\alpha \leq \alpha_{0}$ with $\alpha_{0}$ small enough, the series

$$
\sum_{j \geq 0} \alpha^{j} u^{(j)}
$$

converges in $V$ and is solution of problem (2.7).

More generally, if the right-hand side is any element $\varphi$ of $V^{\prime}$, we solve the problem

$$
\text { Find } u \in V, \quad \forall v \in V, \quad a_{0}^{\mathrm{ap}}(u, v)+\alpha a_{1}^{\mathrm{ap}}(u, v)=\langle\varphi, v\rangle,
$$

by the series

$$
\sum_{j \geq-1} \alpha^{j} u^{(j)}
$$

with $u^{(-1)} \in K_{0}$ the unique solution of

$$
\forall v \in K_{0}, \quad a_{1}^{\mathrm{ap}}(u, v)=\langle\varphi, v\rangle,
$$

$u^{(0)}:=A^{-1}\left(f-\bar{A}_{1} u^{(-1)}, 0\right)$, and $u^{(j)}$ for $j \geq 1$ defined as above.

This proves that the operator $V \ni u \longmapsto\left(v \mapsto a_{0}^{\mathrm{ap}}(u, v)+\alpha a_{1}^{\mathrm{ap}}(u, v)\right) \in V^{\prime}$ is onto as soon as $\alpha \leq \alpha_{0}$. Therefore, it is injective. This ends the proof. 


\subsection{Convergence of the continuous problem with respect to the penalization parameter}

We assume that $a_{0}$ is strongly coercive on $V(\Omega)$. Then the kernel

$$
K_{0}:=\left\{v \in V(\mathcal{D}): \forall u \in V(\mathcal{D}), a_{0}(u, v)=0\right\},
$$

is by definition the space of $v \in H_{0}^{1}(\mathcal{D})$ such that for all $u \in H_{0}^{1}(\mathcal{D})$,

$$
\int_{\Omega} b_{0}(u, v)(x) d x=0 .
$$

Since any function $u \in V(\Omega)$ can be extended in a function $\bar{u} \in H_{0}^{1}(\mathcal{D})$, and, conversely, the restriction of any $v \in H_{0}^{1}(\mathcal{D})$ to $\Omega$ is an element of $V(\Omega)$, the coercivity property of $a_{0}$ implies that

$$
K_{0}=\left\{v \in H_{0}^{1}(\mathcal{D}):\left.v\right|_{\Omega}=0\right\} .
$$

Hence $K_{0}$ is the space of the extensions by zero to $\Omega$ of the elements of $H_{0}^{1}(\mathcal{H})$.

Thus the orthogonal space is

$$
K_{0}^{\perp}=\left\{\varphi \in H^{-1}(\mathcal{D}): \forall v \in K_{0},\langle\varphi, v\rangle=0\right\} .
$$

It is the space of the extensions by zero to $\mathcal{H}$ of the elements of $V(\Omega)^{\prime}$.

We assume that $a_{1}$ is strongly coercive on $H_{0}^{1}(\mathcal{H})$ and define the operator $A$ like in (2.12)-(2.14). Let $f \in K_{0}^{\perp}$. The function $u^{(0)}=u_{0}:=A^{-1}(f, 0)$ is by definition the solution of

Find $u \in H_{0}^{1}(\mathcal{D})$ such that $\forall v \in H_{0}^{1}(\mathcal{D}), \quad a_{0}(u, v)=\langle f, v\rangle \quad$ and $\quad \forall v \in H_{0}^{1}(\mathcal{H}), \quad a_{1}(u, v)=0$.

Let for $k=0,1$ the interior and boundary operators $L_{k}$ and $B_{k}$ be such that

$$
\begin{aligned}
& a_{0}(u, v)=-\int_{\Omega} L_{0} u v d x+\int_{\Sigma} B_{0} u v d \sigma, \quad \forall u, v \in V(\Omega) \text { such that } L_{0} u \in L^{2}(\Omega), \\
& a_{1}(u, v)=-\int_{\mathcal{H}} L_{1} u v d x+\int_{\Sigma} B_{1} u v d \sigma, \quad \forall u, v \in H^{1}(\mathcal{H}) \text { such that } L_{1} u \in L^{2}(\mathcal{H}) .
\end{aligned}
$$

We can see that $\left.u_{0}\right|_{\Omega}$ is the solution $u_{0}^{+}$of the mixed Dirichlet (on $\Gamma$ ) Neumann (on $\Sigma$ ) problem associated with $a_{0}$ on $\Omega$, with right-hand side $f$, and $\left.u_{0}\right|_{\mathcal{H}}$ is the solution $u_{0}^{-}$of the Dirichlet problem

$$
L_{1} u_{0}^{-}=0 \text { in } \mathcal{H} \text { and }\left.u_{0}^{-}\right|_{\Sigma}=\left.u_{0}^{+}\right|_{\Sigma} \text {. }
$$

Note that the next term $u^{(1)}:=-A^{-1}\left(\bar{A}_{1} u^{(0)}, 0\right)$ as defined in the proof of Lemma 1 has the following structure. Let $u_{1}^{+}$and $u_{1}^{-}$its restriction to $\Omega$ and $\mathcal{H}$, respectively. Then $u_{1}^{+}$is solution of the mixed problem

$$
L_{0} u_{1}^{+}=0 \text { in } \Omega,\left.\quad u_{1}^{+}\right|_{\Gamma}=0, \quad \text { and }\left.\quad B_{0} u_{1}^{+}\right|_{\Sigma}=\left.B_{1} u_{0}^{+}\right|_{\Sigma},
$$

and $u_{1}^{-}$is the solution of the Dirichlet problem

$$
L_{1} u_{1}^{-}=0 \text { in } \mathcal{H} \text { and }\left.u_{1}^{-}\right|_{\Sigma}=\left.u_{1}^{+}\right|_{\Sigma}
$$

We have a statement similar to Lemma 1 
Lemma 2 In the continuous framework, let $a_{0}$ be strongly coercive on $V(\Omega)$ and $a_{1}$ be strongly coercive on $H_{0}^{1}(\mathcal{H})$. Let $f \in V(\Omega)^{\prime}$. Then for $\alpha$ small enough, the problem

$$
\text { Find } u_{\alpha} \in V(\mathcal{D}), \quad \forall v \in V(\mathcal{D}), \quad a_{0}\left(u_{\alpha}, v\right)+\alpha a_{1}\left(u_{\alpha}, v\right)=\langle f, v\rangle
$$

has a unique solution. Let $u_{0}$ be the solution of (2.16). Then $u_{\alpha}$ tends to $u_{0}$ in $H^{1}(\mathcal{D})$, and

$$
\left\|u_{\alpha}-u_{0}\right\|_{H^{1}(\mathcal{D})} \leq C \alpha\|f\|_{V(\Omega)^{\prime}} .
$$

Proof: It follows the same lines as the proof of Lemma 1. Since $\varphi \mapsto A^{-1}(\varphi, 0)$ is continuous from $V(\Omega)^{\prime}$ into $H^{1}(\mathcal{D})$, and $u^{(j-1)} \mapsto \bar{A}_{1} u^{(j-1)}$ is continuous from $H^{1}(\mathcal{D})$ into $V(\Omega)^{\prime}$, we have an estimate

$$
\left\|u^{(j)}\right\|_{H^{1}(\mathcal{D})} \leq C\left\|u^{(j-1)}\right\|_{H^{1}(\mathcal{D})}, \quad \forall j \geq 1 .
$$

We deduce the convergence in $H^{1}(\mathcal{D})$ of the series $\sum_{j \geq 0} \alpha^{j} u^{(j)}$ and the estimate of the Lemma.

\section{Céa and Strang lemmas}

We revisit these lemmas to check that strict positiveness of the bilinear forms is not necessary.

Lemma 3 Let a be a real symmetric bilinear form, non-negative on the space $V$ :

$$
\forall u \in V, \quad a(u, u) \geq 0,
$$

defining the semi-norm

$$
|u|_{a}:=a(u, u)^{\frac{1}{2}} .
$$

Let $V^{\text {ap }}$ be a subspace of $V$. Let $f \in V^{\prime}$. We assume that $u \in V$ and $u^{\text {ap }} \in V^{\text {ap }}$ satisfy

$$
a(u, v)=\langle f, v\rangle \quad \forall v \in V \quad \text { and } \quad a\left(u^{\text {ap }}, v^{\text {ap }}\right)=\left\langle f, v^{\text {ap }}\right\rangle \quad \forall v^{\text {ap }} \in V^{\text {ap }} .
$$

Then

$$
\left|u-u^{\mathrm{ap}}\right|_{a} \leq\left|u-v^{\mathrm{ap}}\right|_{a} \quad \forall v^{\mathrm{ap}} \in V^{\mathrm{ap}} .
$$

Proof: We have for all $v^{\text {ap }} \in V^{\text {ap }}$

$$
a\left(u-u^{\mathrm{ap}}, u-u^{\mathrm{ap}}\right)=a\left(u-u^{\mathrm{ap}}, u-v^{\mathrm{ap}}+v^{\mathrm{ap}}-u^{\mathrm{ap}}\right)=a\left(u-u^{\mathrm{ap}}, u-v^{\mathrm{ap}}\right) .
$$

Inequality (3.1) then follows by Cauchy-Schwartz inequality.

Lemma 4 Let a be a real symmetric bilinear form, non-negative on the space $V$. Let $V^{\text {ap }}$ be a subspace of $V$ and let $a^{\text {ap }}$ be a real symmetric bilinear form, non-negative on $V^{\mathrm{ap}}$. Let $d(f, v)$ be a duality pairing between $V^{\prime}$ and $V$, and $d^{\text {ap }}$ be a duality pairing between $V^{\prime}$ and $V^{\text {ap }}$. We define the semi-norms

$$
|u|_{a}:=a(u, u)^{\frac{1}{2}} \quad \text { and } \quad|u|_{a^{\text {ap }}}:=a^{\mathrm{ap}}(u, u)^{\frac{1}{2}} .
$$

We assume that there exists a positive constant $C_{\mathrm{ap}}$ such that

$$
\left|v^{\mathrm{ap}}\right|_{a} \leq C_{\mathrm{ap}}\left|v^{\mathrm{ap}}\right|_{a^{\text {ap }}} \forall v^{\mathrm{ap}} \in V^{\mathrm{ap}} .
$$


We assume that $u \in V$ and $u^{\text {ap }} \in V^{\text {ap }}$ satisfy

$$
a(u, v)=d(f, v) \quad \forall v \in V \quad \text { and } \quad a^{\mathrm{ap}}\left(u^{\mathrm{ap}}, v^{\mathrm{ap}}\right)=d^{\mathrm{ap}}\left(f, v^{\mathrm{ap}}\right) \quad \forall v^{\mathrm{ap}} \in V^{\mathrm{ap}} .
$$

Then for all $v^{\mathrm{ap}} \in V^{\mathrm{ap}}$ the following two inequalities hold:

$$
\left|u-u^{\mathrm{ap}}\right|_{a} \leq\left(1+C_{\mathrm{ap}}^{2}\right)\left|u-v^{\mathrm{ap}}\right|_{a}+C_{\mathrm{ap}}^{2}\left\{\sup _{w^{\mathrm{ap}} \in V^{\mathrm{ap}}} \frac{\left(a-a^{\mathrm{ap}}\right)\left(v^{\mathrm{ap}}, w^{\mathrm{ap}}\right)}{\left|w^{\mathrm{ap}}\right|_{a}}+\sup _{w^{\mathrm{ap}} \in V^{\mathrm{ap}}} \frac{\left(d-d^{\mathrm{ap}}\right)\left(f^{\mathrm{ap}}, w^{\mathrm{ap}}\right)}{\left|w^{\mathrm{ap}}\right|_{a}}\right\}
$$

and

$$
\left|u-u^{\mathrm{ap}}\right|_{a} \leq\left(1+C_{\mathrm{ap}}^{2}\right)\left|u-v^{\mathrm{ap}}\right|_{a}+C_{\mathrm{ap}}\left\{\sup _{w^{\mathrm{ap}} \in V^{\mathrm{ap}}} \frac{\left(a-a^{\mathrm{ap}}\right)\left(v^{\mathrm{ap}}, w^{\mathrm{ap}}\right)}{\left|w^{\mathrm{ap}}\right|_{a^{\text {ap }}}}+\sup _{w^{\mathrm{ap}} \in V^{\mathrm{ap}}} \frac{\left(d-d^{\mathrm{ap}}\right)\left(f^{\mathrm{ap}}, w^{\mathrm{ap}}\right)}{\left|w^{\mathrm{ap}}\right|_{a^{\mathrm{ap}}}}\right\}
$$

Proof: Let us choose $v^{\text {ap }} \in V^{\text {ap }}$. We write

$$
\left|u-u^{\mathrm{ap}}\right|_{a} \leq\left|u-v^{\mathrm{ap}}\right|_{a}+\left|v^{\mathrm{ap}}-u^{\mathrm{ap}}\right|_{a} .
$$

We set $w^{\mathrm{ap}}=u^{\mathrm{ap}}-v^{\mathrm{ap}}$. Then we evaluate $\left|v^{\mathrm{ap}}-u^{\mathrm{ap}}\right|_{a^{\mathrm{ap}}}^{2}=a^{\mathrm{ap}}\left(v^{\mathrm{ap}}-u^{\mathrm{ap}}, v^{\mathrm{ap}}-u^{\mathrm{ap}}\right)$ :

$$
\left|v^{\mathrm{ap}}-u^{\mathrm{ap}}\right|_{a^{\mathrm{ap}}}^{2}=a^{\mathrm{ap}}\left(u^{\mathrm{ap}}, w^{\mathrm{ap}}\right)-a\left(v^{\mathrm{ap}}, w^{\mathrm{ap}}\right)+\left(a-a^{\mathrm{ap}}\right)\left(v^{\mathrm{ap}}, w^{\mathrm{ap}}\right) .
$$

We note that

$$
a^{\mathrm{ap}}\left(u^{\mathrm{ap}}, w^{\mathrm{ap}}\right)=d^{\mathrm{ap}}\left(f, w^{\mathrm{ap}}\right)=d\left(f, w^{\mathrm{ap}}\right)-\left(d-d^{\mathrm{ap}}\right)\left(f, w^{\mathrm{ap}}\right) .
$$

thus

$$
a^{\mathrm{ap}}\left(u^{\mathrm{ap}}, w^{\mathrm{ap}}\right)=a\left(u, w^{\mathrm{ap}}\right)-\left(d-d^{\mathrm{ap}}\right)\left(f, w^{\mathrm{ap}}\right) .
$$

Combining (3.6) and (3.7):

$$
\left|v^{\mathrm{ap}}-u^{\mathrm{ap}}\right|_{a^{\mathrm{ap}}}^{2}=a\left(u, w^{\mathrm{ap}}\right)-a\left(v^{\mathrm{ap}}, w^{\mathrm{ap}}\right)-\left(d-d^{\mathrm{ap}}\right)\left(f, w^{\mathrm{ap}}\right)+\left(a-a^{\mathrm{ap}}\right)\left(v^{\mathrm{ap}}, w^{\mathrm{ap}}\right) .
$$

Identity (3.8) implies the inequality

$$
\left|v^{\mathrm{ap}}-u^{\mathrm{ap}}\right|_{a^{\text {ap }}}\left|w^{\mathrm{ap}}\right|_{a^{\mathrm{ap}}} \leq\left[\left|u-v^{\mathrm{ap}}\right|_{a}\left|w^{\mathrm{ap}}\right|_{a}+\left|\left(d-d^{\mathrm{ap}}\right)\left(f, w^{\mathrm{ap}}\right)\right|+\left|\left(a-a^{\mathrm{ap}}\right)\left(v^{\mathrm{ap}}, w^{\mathrm{ap}}\right)\right|\right] .
$$

Combining (3.9) with (3.2):

$$
\left|v^{\mathrm{ap}}-u^{\mathrm{ap}}\right|_{a}\left|w^{\mathrm{ap}}\right|_{a} \leq C_{\mathrm{ap}}^{2}[\text { RHS of (3.9)]. }
$$

Finally, we deduce (3.3) by dividing (3.10) by $\left|w^{\mathrm{ap}}\right|_{a}$, taking the sup in $w^{\mathrm{ap}} \in V^{\mathrm{ap}}$, and coming back to (3.5).

The second estimate (3.4) is obtained by dividing (3.9) by $\left|w^{\mathrm{ap}}\right|_{a^{\text {ap }}}$ and using (3.2) next.

We can use Lemma 4 with $a=a_{0}$ or $a=a_{0}+\alpha a_{1}$, and also take numerical integration into account in $a^{\text {ap }}$ and $d^{\text {ap }}$. 
Assuming exact integration, we can also use the lemma with $a=a_{0}, a^{\text {ap }}=a_{0}+\alpha a_{1}$ and $d=d^{\text {ap }}$. In this case, the third term in the right-hand side of (3.4) is zero and the second one is the sup for $w^{\text {ap }} \in V^{\text {ap }}$ of

$$
\frac{\left(a-a^{\mathrm{ap}}\right)\left(v^{\mathrm{ap}}, w^{\mathrm{ap}}\right)}{\left|w^{\mathrm{ap}}\right|_{a^{\mathrm{ap}}}}=\frac{\alpha a_{1}\left(v^{\mathrm{ap}}, w^{\mathrm{ap}}\right)}{\left|w^{\mathrm{ap}}\right|_{a^{\mathrm{ap}}}} \leq \frac{\alpha\left|v^{\mathrm{ap}}\right|_{a_{1}}\left|w^{\mathrm{ap}}\right|_{a_{1}}}{\left(\left|w^{\mathrm{ap}}\right|_{a_{0}}^{2}+\alpha\left|w^{\mathrm{ap}}\right|_{a_{1}}^{2}\right)^{1 / 2}} \leq \sqrt{\alpha}\left|v^{\mathrm{ap}}\right|_{a_{1}} .
$$

In this case, $C_{\mathrm{ap}}=1$ and (3.4) yields:

Corollary 5 Under the conditions of Lemma 4 with $a=a_{0}$ and $a^{\text {ap }}=a_{0}+\alpha a_{1}$, we assume moreover exact integration $\left(d=d^{\mathrm{ap}}\right)$. Then we have the estimate

$$
\left|u-u_{\alpha}^{\mathrm{ap}}\right|_{a_{0}} \leq 2\left|u-v^{\mathrm{ap}}\right|_{a_{0}}+\sqrt{\alpha}\left|v^{\mathrm{ap}}\right|_{a_{1}} \quad \forall v^{\mathrm{ap}} \in V^{\mathrm{ap}} .
$$

Remark 6 Under the conditions of Lemma 3 with $a=a_{0}$ and Lemma 1 with $a_{0}^{\text {ap }}=a_{0}$ and $a_{1}^{\text {ap }}=a_{1}$ (i.e., assuming exact integration), we can write for all $\alpha$

$$
\left|u-u_{\alpha}^{\mathrm{ap}}\right|_{a_{0}} \leq\left|u-u_{0}^{\mathrm{ap}}\right|_{a_{0}}+\left|u_{0}^{\mathrm{ap}}-u_{\alpha}^{\mathrm{ap}}\right|_{a_{0}} .
$$

Lemma 3 yields that $\left|u-u_{0}^{\mathrm{ap}}\right|_{a_{0}}$ is less than $\left|u-v^{\text {ap }}\right|_{a_{0}}$ for all $v^{\text {ap }} \in V^{\text {ap }}$ and Lemma 1 yields the information that $\left|u_{0}^{\mathrm{ap}}-u_{\alpha}^{\mathrm{ap}}\right|_{a_{0}}$ is a $\mathcal{O}(\alpha)$. But the multiplicative constant in front of $\alpha$ a priori depends on the discretization. That is why we cannot improve estimate (3.11) by replacing $\sqrt{\alpha}$ with $\alpha$, in general. In fact, our numerical experiments also display a $\sqrt{\alpha}$ behavior in the general case.

\section{$4 p$-version of finite elements}

Let $\mathcal{T}$ be a fixed mesh of the domain $\mathcal{D}$ and let $V_{p}(\mathcal{D})$ be the $p$-extension over the mesh $\mathcal{T}$ with the boundary condition $v=0$ on $\Gamma$. We denote by

$$
\Omega^{\mathrm{ap}}=\operatorname{interior}\left\{\bigcup_{K \in \mathcal{T} \mid K \cap \Omega \neq \emptyset} \bar{K}\right\} \text { and } \mathcal{H}^{\mathrm{ap}}=\mathcal{D} \backslash \bar{\Omega}^{\mathrm{ap}} .
$$

We note that $\Gamma$ is contained in $\partial \Omega^{\mathrm{ap}}$ and we denote the common boundary $\partial \Omega^{\mathrm{ap}} \cap \partial \mathcal{H}^{\mathrm{ap}}$ by $\Gamma^{\mathrm{ap}}$.

We denote by $\mathcal{A}(\overline{\mathcal{U}})$ the space of analytic functions up to the boundary of the domain $\mathcal{U}$.

Theorem 7 Let $a_{0}$ be strongly coercive on $V(\Omega)$ and $a_{1}$ be strongly coercive on $H_{0}^{1}(\mathcal{H})$. Let $f \in \mathcal{A}(\bar{\Omega})$. Let $u_{0}$ be the solution of the mixed problem (2.5). We assume that $u_{0}$ admits an analytic extension $\bar{u}_{0} \in \mathcal{A}\left(\bar{\Omega}^{\mathrm{ap}}\right)$. For $\alpha>0$ and any $p \geq 1$, let $u^{\mathrm{ap}}[p, \alpha]$ be the solution of problem $(2.7)$ with $V^{\mathrm{ap}}(\mathcal{D})=V_{p}$ and assuming exact integration. Then there exist $c>0$ and $\gamma>0$ such that for all $\alpha>0$ small enough and all $p \geq 1$

$$
\left\|u_{0}-u^{\mathrm{ap}}[p, \alpha]\right\|_{H^{1}(\Omega)} \leq c\left(\mathrm{e}^{-p \gamma}+\sqrt{\alpha}\right) .
$$


Proof: We use Corollary 5. By the coercivity assumption on $a_{0}$, we find that the semi-norm $|\cdot|_{a_{0}}$ is equivalent to the $H^{1}(\Omega)$-norm.

Thus, relying on estimate (3.11), it suffices to find $v^{\text {ap }} \in V_{p}$ such that

$$
\left\|u_{0}-v^{\mathrm{ap}}\right\|_{H^{1}(\Omega)} \leq c \mathrm{e}^{-p \gamma} \text { and }\left\|v^{\mathrm{ap}}\right\|_{H^{1}(\mathcal{H})} \leq c .
$$

Since $\bar{u}_{0} \in \mathcal{A}\left(\bar{\Omega}^{\mathrm{ap}}\right)$, we can find $v_{p} \in V_{p}\left(\Omega^{\mathrm{ap}}\right)$ such that

$$
\left\|\bar{u}_{0}-v_{p}\right\|_{H^{1}\left(\Omega^{\mathrm{ap}}\right)} \leq c_{0} \mathrm{e}^{-p \gamma} .
$$

Therefore, in particular,

$$
\left\|v_{p}\right\|_{H^{\frac{1}{2}}\left(\Gamma^{\mathrm{ap}}\right)} \leq c_{1}
$$

There exists $\tilde{v}_{p} \in V_{p}\left(\mathcal{H}^{\text {ap }}\right)$ such that

$$
\left.\tilde{v}_{p}\right|_{\Sigma}=\left.v_{p}\right|_{\Sigma} \text { and } \quad\left\|\tilde{v}_{p}\right\|_{H^{1}\left(\mathcal{H}^{\text {ap }}\right)} \leq c_{2} .
$$

We define $v^{\text {ap }}$ by $v_{p}$ on $\Omega^{\text {ap }}$ and $\tilde{v}_{p}$ on $\mathcal{H}^{\text {ap }}$ and we deduce (4.3) from (4.4)-(4.6).

In the case where the grid is matching with the interface $\Sigma$, the estimate (4.2) is improved (see also Remark 6 on this question - why such an improvement does not hold in the general case).

Theorem 8 Under the assumptions of Theorem 7 we assume moreover that $\Omega^{\mathrm{ap}}=\Omega$. Then there exist $c>0$ and $\gamma>0$ such that for all $\alpha>0$ small enough and all $p \geq 1$

$$
\left\|u_{0}-u^{\mathrm{ap}}[p, \alpha]\right\|_{H^{1}(\Omega)} \leq c\left(\mathrm{e}^{-p \gamma}+\alpha\right) .
$$

Proof: Let us choose the degree $p$. When the grid is matching the interface $\Sigma$, the terms $u^{(j)}[p]$ of the expansion of $u^{\mathrm{ap}}[p, \alpha]$ in powers of $\alpha$ can be described as discrete FEM solutions: Let $u_{j}^{+}[p]$ and $u_{j}^{-}[p]$ be the restrictions of $u^{(j)}[p]$ to $\Omega=\Omega^{\text {ap }}$ and $\mathcal{H}=\mathcal{H}^{\text {ap }}$, respectively. For $j \geq 1, u_{j}^{+}[p]$ is the discrete solution of the mixed problem in $\Omega$ with Neumann data on $\Sigma$ coming from $u_{j-1}^{-}[p]$, and $u_{j}^{-}[p]$ is the discrete solution of the Dirichlet problem in $\mathcal{H}$ with Dirichlet data on $\Sigma$ coming from $u_{j}^{+}[p]$. The uniformity of continuity constants with respect to $p$ can be deduced.

If $\Omega^{\text {ap }}$ coincides with $\mathcal{D}$, we may even have exponential convergence with $\alpha=0$ :

Theorem 9 We assume that $\Omega^{\mathrm{ap}}=\mathcal{D}$ (i.e., $\mathcal{H}^{\mathrm{ap}}=\emptyset$ ). Let $a_{0}$ be strongly coercive on $V(\Omega)$. Let $f \in \mathcal{A}(\bar{\Omega})$ and let $u_{0}$ be the solution of the mixed problem (2.5). We assume that $u_{0}$ admits an analytic extension $\bar{u}_{0} \in \mathcal{A}\left(\bar{\Omega}^{\mathrm{ap}}\right)$. Let $u^{\mathrm{ap}}[p]$ be solution of (here we assume exact integration)

$$
\text { Find } u^{\mathrm{ap}} \in V_{p}(\mathcal{D}), \quad \forall v \in V_{p}(\mathcal{D}), \quad a_{0}\left(u^{\mathrm{ap}}, v\right)=\int_{\mathcal{D}} f(x) v(x) d x,
$$

Then there exist $c>0$ and $\gamma>0$ such that for all $p \geq 1$

$$
\left\|u_{0}-u^{\mathrm{ap}}[p]\right\|_{H^{1}(\Omega)} \leq c \mathrm{e}^{-p \gamma} .
$$


Proof: This is a consequence of Céa Lemma 3 if we have proved that $u^{\text {ap }}[p]$ does exist. Let us choose the degree $p$. If suffices to show that the kernel $K_{0}$ defined as

$$
K_{0}=\left\{v \in V_{p}(\mathcal{D}): \forall u \in V_{p}(\mathcal{D}), a_{0}(u, v)=0\right\}
$$

is reduced to $\{0\}$. Let $v \in K_{0}$. Then $a_{0}(v, v)=0$. Since $\left.v\right|_{\Omega}$ belongs to $V(\Omega)$, we deduce from the coercivity property of $a_{0}$ that $\left.v\right|_{\Omega} \equiv 0$. By assumption any element $K$ of the mesh $\mathcal{T}$ has a non-empty intersection with $\Omega$. Since $v$ is a polynomial on $K$ which is zero on $K \cap \Omega$, it is zero over the whole of $K$. Hence $v \equiv 0$, which ends the proof.

The difficulty is that in practice, the assumption $\bar{u}_{0} \in \mathcal{A}\left(\bar{\Omega}^{\mathrm{ap}}\right)$ has no reason to be satisfied in general. Nevertheless, we will see examples where this assumption is not satisfied, and where exponential convergence for $\alpha=0$ can be observed.

\section{1D test problem with Neumann boundary conditions at the hole}

\subsection{Problem definition and exact solution}

As the simplest possible model for a hole in 1D, we choose the two component domain

$$
\Omega=\left(-1,-\frac{1}{4}\right) \cup\left(\frac{1}{4}, 1\right) .
$$

Thus, the "hole" $\mathcal{H}$ is the interval $\left(-\frac{1}{4}, \frac{1}{4}\right)$. The associated fictitious domain $\mathcal{D}$ is

$$
\mathcal{D}=(-1,1)
$$

We consider the family of bilinear forms, indexed by the coefficient $\alpha$

$$
a_{\alpha}(u, v)=\int_{-1}^{-1 / 4} u^{\prime} v^{\prime}+k^{2} u v d x+\int_{1 / 4}^{1} u^{\prime} v^{\prime}+k^{2} u v d x+\alpha \int_{-1 / 4}^{1 / 4} u^{\prime} v^{\prime} d x
$$

Here, the coefficient $\alpha$ inside the hole has been set to zero only for the mass matrix and small for the stiffness matrix. It corresponds to our general setting with

$$
b_{0}(u, v)=u^{\prime} v^{\prime}+k^{2} u v \quad \text { and } \quad b_{1}(u, v)=u^{\prime} v^{\prime} .
$$

The domain $\Omega$ is symmetric with respect to the origin. We investigate two problems with different symmetry properties: the first one is odd, and the second, even.

The odd problem is described by

$$
\left\{\begin{aligned}
-u^{\prime \prime}(x)+k^{2} u(x) & =0, \quad x \in \Omega \\
u(-1) & =-1 \\
u(1) & =1 \\
u^{\prime}\left(-\frac{1}{4}\right) & =0 \\
u^{\prime}\left(\frac{1}{4}\right) & =0
\end{aligned}\right.
$$


with the corresponding exact solution

$$
u(x)= \begin{cases}\frac{e^{k(0.5-x)}+e^{k x}}{e^{-0.5 k}+e^{k}} & \text { if } \quad x>0, \\ -\frac{e^{k(0.5+x)}+e^{-k x}}{e^{-0.5 k}+e^{k}} & \text { if } \quad x<0 .\end{cases}
$$

The even problem reads

where

$$
\left\{\begin{aligned}
-u^{\prime \prime}(x)+k^{2} u(x) & =0, \quad x \in \Omega \\
u(-1) & =1 \\
u(1) & =1 \\
u^{\prime}\left(-\frac{1}{4}\right) & =0 \\
u^{\prime}\left(\frac{1}{4}\right) & =0
\end{aligned}\right.
$$

denotes the exact solution.

$$
u(x)=\left\{\begin{array}{lll}
\frac{e^{k(0.5-x)}+e^{k x}}{e^{-0.5 k}+e^{k}} & \text { if } \quad x>0 \\
\frac{e^{k(0.5+x)}+e^{-k x}}{e^{-0.5 k}+e^{k}} & \text { if } \quad x<0
\end{array}\right.
$$

\subsection{Finite cell approach}

The bilinear form (5.3) as described in the previous subsection is discretized by means of the finite cell method. To this end, the fictitious domain $\mathcal{D}$ is subdivided into a mesh $\mathcal{T}$ consisting of $n_{c}$ cells with the corresponding nodal coordinates denoted as $X_{c}, X_{c+1}$. On each cell hierarchic shape functions $N_{i}$ based on integrated Legendre polynomials $[25,4]$ are applied to discretize the trial and test functions. The discretization of the bilinear form results in a matrix composed of two parts: the stiffness matrix and the mass matrix. Since in general the cells do not conform with the geometry, the integrand of the cell stiffness matrix

$$
K_{i j}^{c}=\int_{X_{c}}^{X_{c+1}} \alpha \frac{d N_{i}}{d x} \frac{d N_{j}}{d x} d x=\frac{2}{X_{c+1}-X_{c}} \int_{-1}^{1} \alpha \frac{d N_{i}}{d \xi} \frac{d N_{j}}{d \xi} d \xi, \quad i, j=1,2,3, \ldots
$$

and the cell mass matrix

$$
M_{i j}^{c}=\int_{X_{c}}^{X_{c+1}} \alpha_{M} k^{2} N_{i} N_{j} d x=\frac{X_{c+1}-X_{c}}{2} \int_{-1}^{1} \alpha_{M} k^{2} N_{i} N_{j} d \xi, \quad i, j=1,2,3, \ldots
$$

might be discontinuous. In (5.8) and (5.9) $x, \xi$ denote the global and local coordinates, which are related to each other by a linear mapping function. Note, that we distinguish between $\alpha$ and $\alpha_{M}$. In (5.8) $\alpha$ corresponds to Equation (5.3), whereas $\alpha_{M}=0$ in (5.9) assures that inside the hole there is no contribution of the mass matrix. The integration of the cell matrices is carried out by applying a composed Gaussian quadrature. To account for the hole, i.e. the jump of $\alpha, \alpha_{M}$, the corresponding cell is divided for the purpose of (exact) integration into $n_{s c}$ sub-cells, so that on each sub-cell $\alpha$ and $\alpha_{M}$ are constant. In this way it is possible to perform an exact computation of the stiffness and mass matrix with $n_{G}=p+1$ Gaussian points applied on the sub-cells which are introduced just for integration purposes. Considering a mesh with one cell only, the minimum number of sub-cells needed for an exact integration is $n_{s c}=3$. 


\subsection{Evaluation of the error}

In order to quantify the efficiency and accuracy of the finite cell method we briefly present in this section the definition of the error. Thanks to availability of the exact solution, the error

$$
e=u-u^{\mathrm{ap}}
$$

of the finite cell approximation can be evaluated directly. In the following we compute the error in the $H^{1}$ norm

$$
\|e\|_{H^{1}}^{2}=\int_{-1}^{-1 / 4}\left(e^{\prime 2}+k^{2} e^{2}\right) d x+\int_{1 / 4}^{1}\left(e^{\prime 2}+k^{2} e^{2}\right) d x
$$

by considering the contribution in the domain $\Omega$ only, i.e. ignoring the results of the finite cell method in the hole $\mathcal{H}$. Since the computation of the error in $H^{1}$ norm (5.11) involves the integration of nonpolynomials a Gaussian quadrature will not yield exact values. Therefore we apply an composed Gaussian quadrature as described in the previous section in order reliably determine the error.

\subsection{Numerical examples for Neumann boundary conditions at the hole}

In the following we present several numerical results obtained with the finite cell method discretizing the problem described in Section 5.1. We choose $k=3$ and compute the error in terms of Equation (5.11).

\subsubsection{Non-matching grid with one cell}

First, we choose one finite cell with nodal coordinates $X_{1}=0$ and $X_{2}=2$ to discretize the fictitious domain and perform a $p$-extension with $p=1,2,3, \ldots, 20$. In this example, $\alpha$ and likewise $\alpha_{M}$ is set to 0 inside the hole. The integration of the stiffness and mass matrix is carried out exactly. A comparison of the exact solution and the finite cell approximation for $p=20$ is given in Figure 1. It can be seen that one cell very accurately represents the exact solution. Note that in the hole the FCM approximation presents a smooth behaviour, connecting the two branches of the exact solution. To quantify the efficiency more precisely, the error $\|e\|_{H^{1}}^{2}$ of the FCM approximation with $p=1,2,3, \ldots$ is plotted in Figure 2 against the polynomial degree. From this it is evident that an exponential convergence can be obtained although the mesh consisting of one cell only does not conform to the geometry. It is also noted that the convergence of the problem with the even solution is faster.

Based on the Céa Lemma 3 used with the form $a_{0}$, we prove this exponential convergence if we know the existence of a polynomial $v_{p}$ of degree $\leq p$ such that

$$
\left\|u-v_{p}\right\|_{H^{1}\left(-1,-\frac{1}{4}\right)}+\left\|u-v_{p}\right\|_{H^{1}\left(\frac{1}{4}, 1\right)} \leq c \mathrm{e}^{-p \gamma} .
$$

Lemma 10 Let $\lambda \in(0,1)$. Let $g$ be a function defined and analytic on the union of intervals $[-1,-\lambda] \cup$ $[\lambda, 1]$. There exists $c>0$ and $\gamma>0$ and for all $p \geq 1$ a polynomial $v_{p} \in \mathbb{P}_{p}(-1,1)$ such that

$$
\left\|g-v_{p}\right\|_{H^{1}\left(-1,-\frac{1}{4}\right)}+\left\|g-v_{p}\right\|_{H^{1}\left(\frac{1}{4}, 1\right)} \leq c \mathrm{e}^{-p \gamma} .
$$



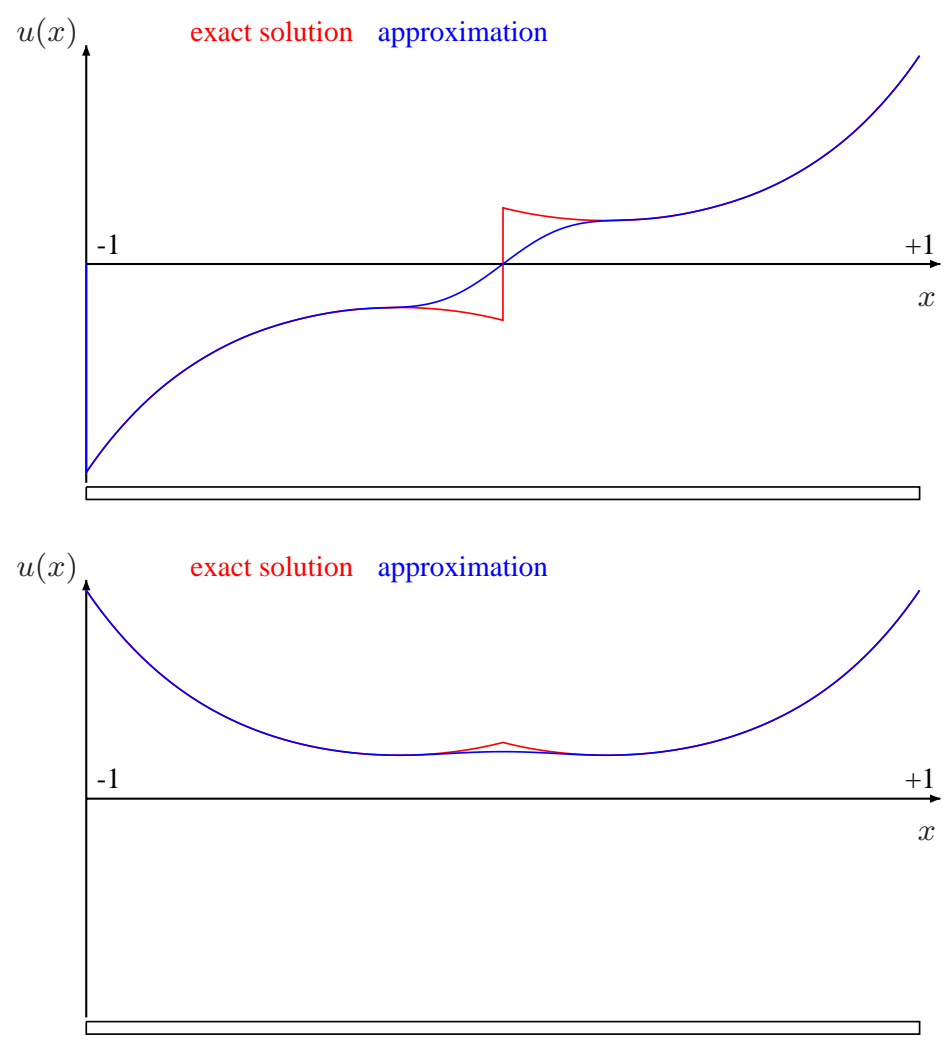

Figure 1: Comparison of exact solution and FCM approximation with one cell and $p=20$; odd problem (upper part), even problem (lower part)

Proof: Considering the even and odd parts of $g$, we may reduce to the case when $g$ is either even or odd.

Even case: By the formula $G(t)=g(\sqrt{t})$ we define an analytic function $G \in \mathcal{A}[\sqrt{\lambda}, 1]$ such that

$$
\forall x \in[-1,-\lambda] \cup[\lambda, 1], \quad g(x)=G\left(x^{2}\right) .
$$

Let $p=2 q$ be a positive (even) integer. There exists $\Phi_{q} \in \mathbb{P}_{q}(\sqrt{\lambda}, 1)$ satisfying the estimate

$$
\left\|G-\Phi_{q}\right\|_{H^{1}(\sqrt{\lambda}, 1)} \leq c \mathrm{e}^{-q \gamma^{\prime}} .
$$

We set $v_{p}(x)=\Phi_{q}\left(x^{2}\right)$ and have proved (5.13) with $\gamma=\gamma^{\prime} / 2$.

Odd case: Similarly, by the formula $G(t)=t^{-1 / 2} g(\sqrt{t})$ we define an analytic function $G \in \mathcal{A}[\sqrt{\lambda}, 1]$ such that

$$
\forall x \in[-1,-\lambda] \cup[\lambda, 1], \quad g(x)=x G\left(x^{2}\right) .
$$

Let $p=2 q+1$ be a positive (odd) integer. There exists $\Phi_{q} \in \mathbb{P}_{q}(\sqrt{\lambda}, 1)$ satisfying the estimate (5.14). We set $v_{p}(x)=x \Phi_{q}\left(x^{2}\right)$ and have proved (5.13) as before. 


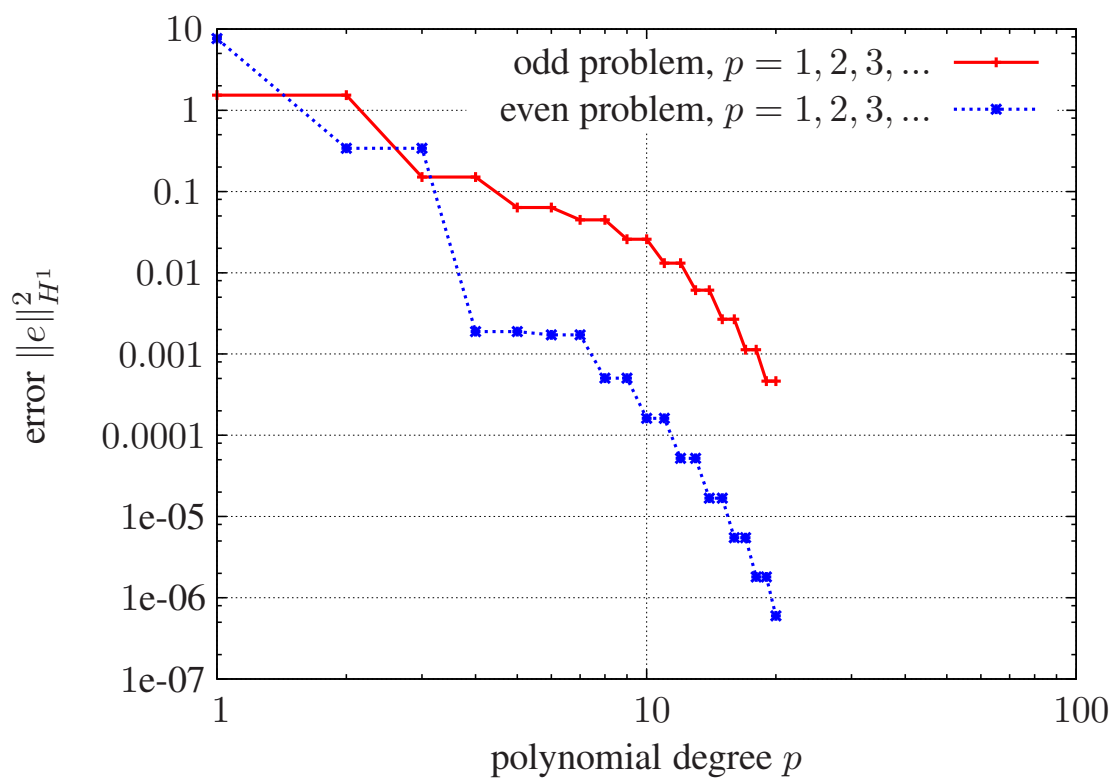

Figure 2: $p$-Extension on mesh with one element, $\alpha=0$, exact integration 


\subsubsection{Matching grid with three cells}

Next, we consider the odd problem discretized by a mesh with three cells where the layout is such that the hole is precisely covered by one cell. The nodal coordinates $X_{c}$ of the mesh correspond to $\{0,0.75,1.25,2\}$. In this case, the integration of the cell matrices can be carried out exactly by a standard Gaussian quadrature with $n_{G}=p+1$ without the necessity of introducing sub-cells. The aim of this example is to consider the influence of the parameter $\alpha$. In Figure 3 the results of the FCM obtained with $p=20$ for two different values of $\alpha$, i.e. $\alpha=10^{-01}$ and $\alpha=10^{-14}$ are presented. Small deviations from the exact solution are observed in the case of $\alpha=10^{-01}$. These deviations are due to the fact that a value different from $\alpha=0$ inside the hole corresponds to a modification of the original problem, replacing the hole by a (very) soft material. Therefore, it can not be expected that a $p$-extension of the FCM converges to the exact solution of the problem when $\alpha \neq 0$ inside the hole. In order to study the
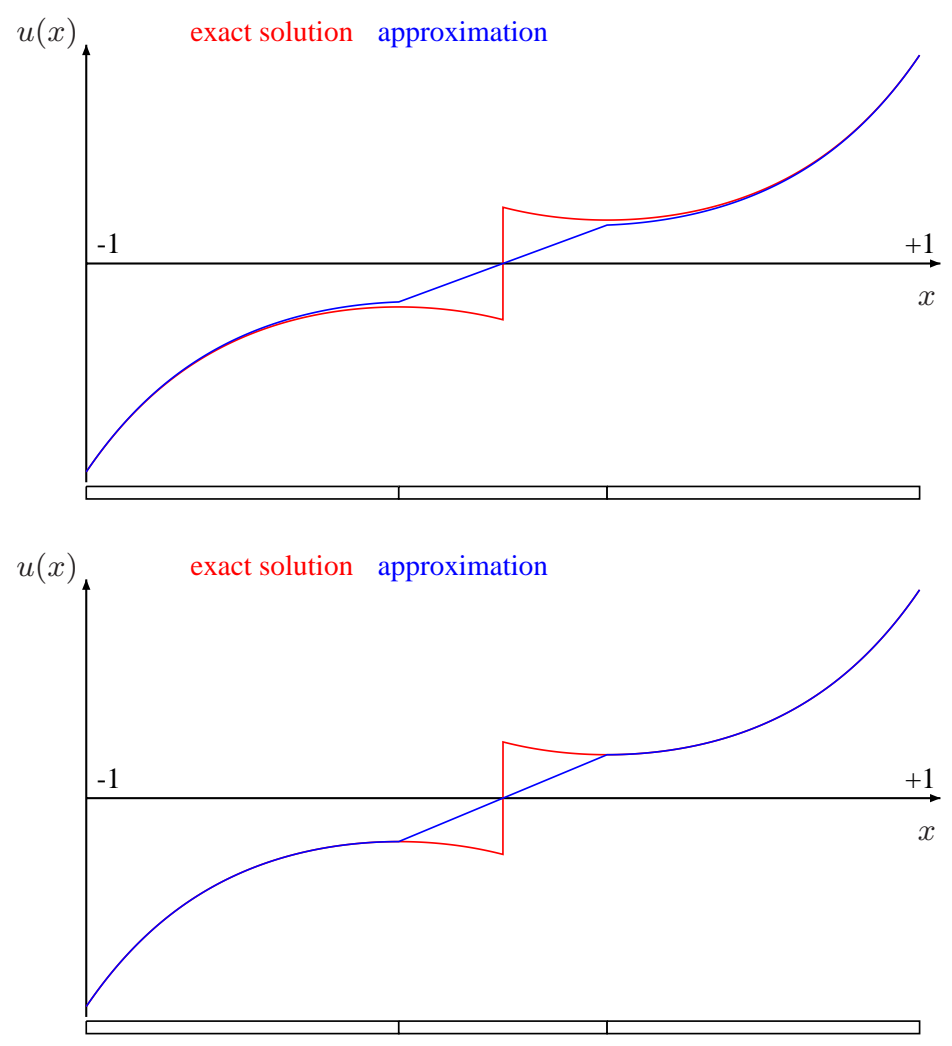

Figure 3: Comparison of exact solution and FCM approximation with $p=20$ and $\alpha=10^{-01}$ (upper part) and $\alpha=10^{-14}$ (lower part)

influence of $\alpha$ let us consider the convergence of a $p$-extension for different values of $\alpha$ inside the hole, see Figure 4. It can be observed that the error $\|e\|_{H^{1}}^{2}$ converges exponentially down to a certain threshold which depends on the value of $\alpha$. The smaller we choose $\alpha$ the higher is the achievable accuracy. The influence of $\alpha$ is investigated more systematically in Figure 5, where the error $\|e\|_{H^{1}}^{2}$ is plotted against $\alpha$ obtained with three cells with a polynomial degree of $p=20$. From this it can be observed that a $p$-extension on the mesh with three cells, with nodes being aligned to the hole, yields exponential convergence up to the error $\alpha^{2}$ in quadratic energy. This is in coherence with Theorem 8. 


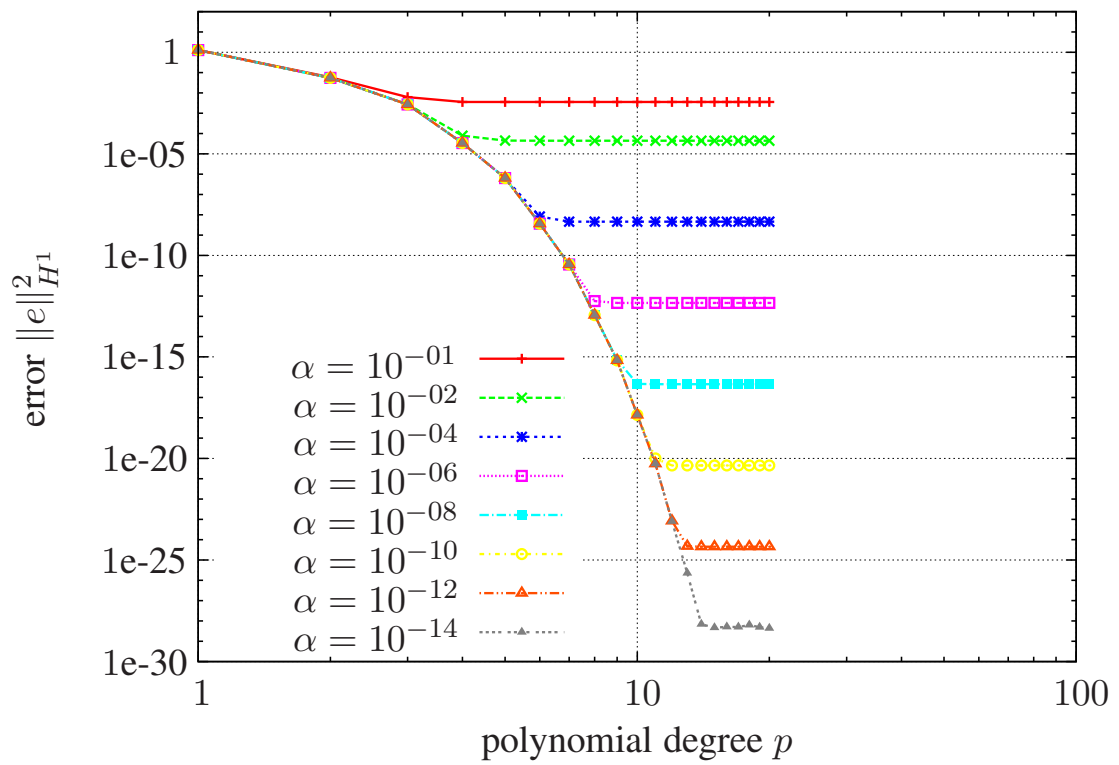

Figure 4: $p$-Extension on a mesh with three cells for different values of $\alpha$

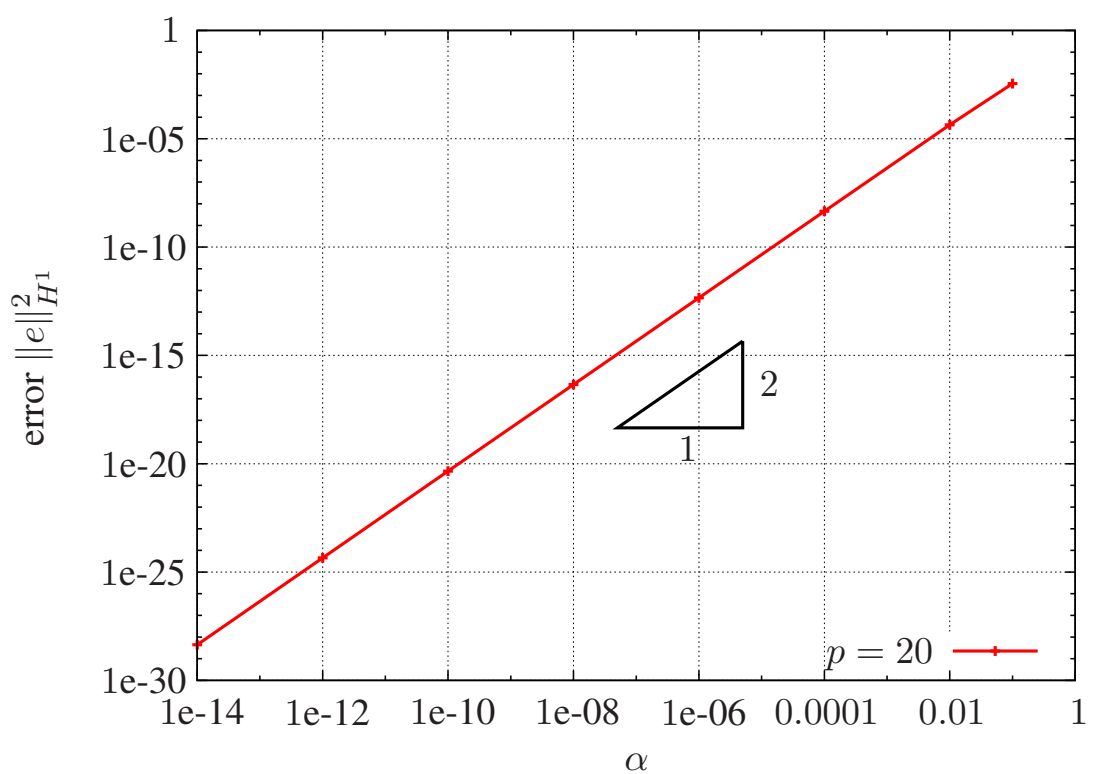

Figure 5: Influence of $\alpha$ on the error 


\subsubsection{Non-matching grid with three cells - case I}

Again, we consider a mesh with three cells but this time non-matching with the hole. The nodal coordinates $X_{c}$ of the mesh correspond to $\{0,0.7,1.3,2\}$. Therefore the nodes of the middle element are slightly outside of the hole, or in other words the hole is located complete inside the middle element. A comparison of the exact solution with the FCM approximation with $p=20$ and $\alpha=10^{-14}$ is presented in Figure 6. The convergence of a $p$-extension applying the FCM for different values of $\alpha$ shows again

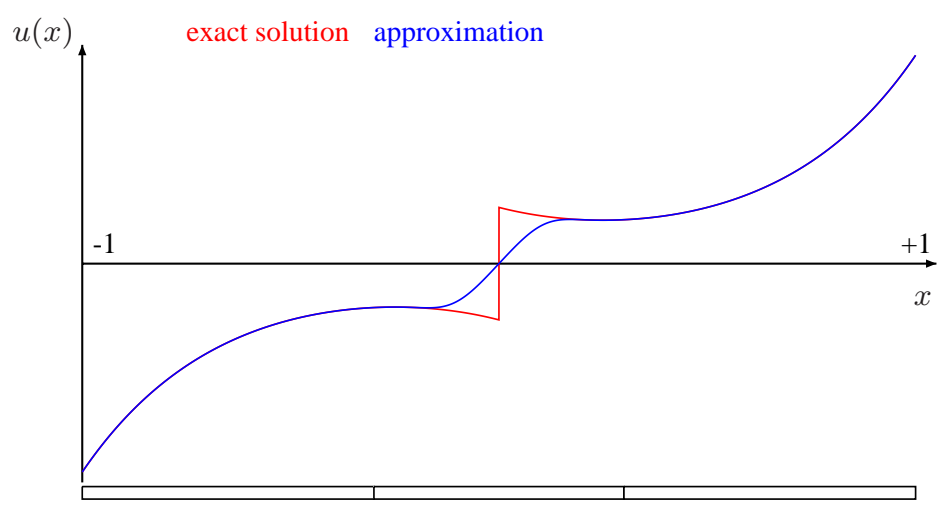

Figure 6: Comparison of exact solution and FCM approximation with $p=20$ and $\alpha=10^{-14}$

an exponential convergence up to a certain threshold depending on the chosen value of $\alpha$. However, in this example where the grid is not matching with the hole, the convergence is not as fast as in the case of the matching grid. The convergence of the error $\|e\|_{H^{1}}^{2}$ with respect to $\alpha$ is plotted in Figure 8 . From

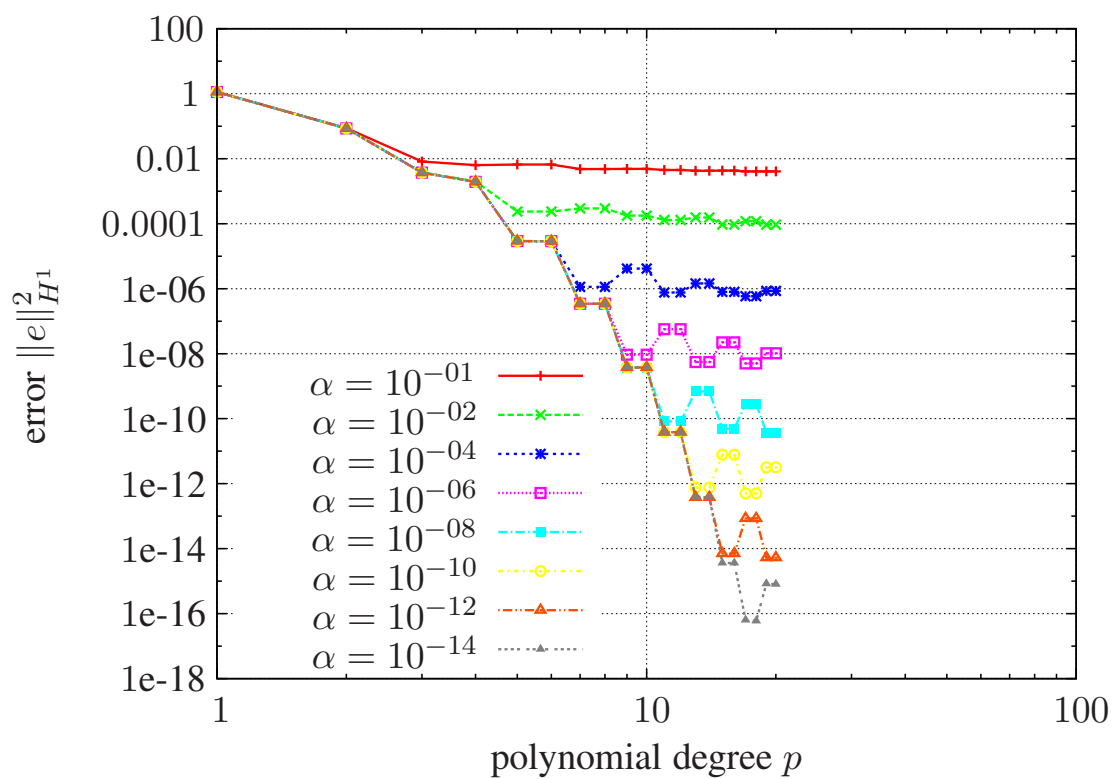

Figure 7: $p$-Extension on mesh with three elements for different $\alpha$ values

this it is evident that the error in quadratic energy depends linearly on $\alpha$. This numerical result is in 
coherence with Theorem 7.

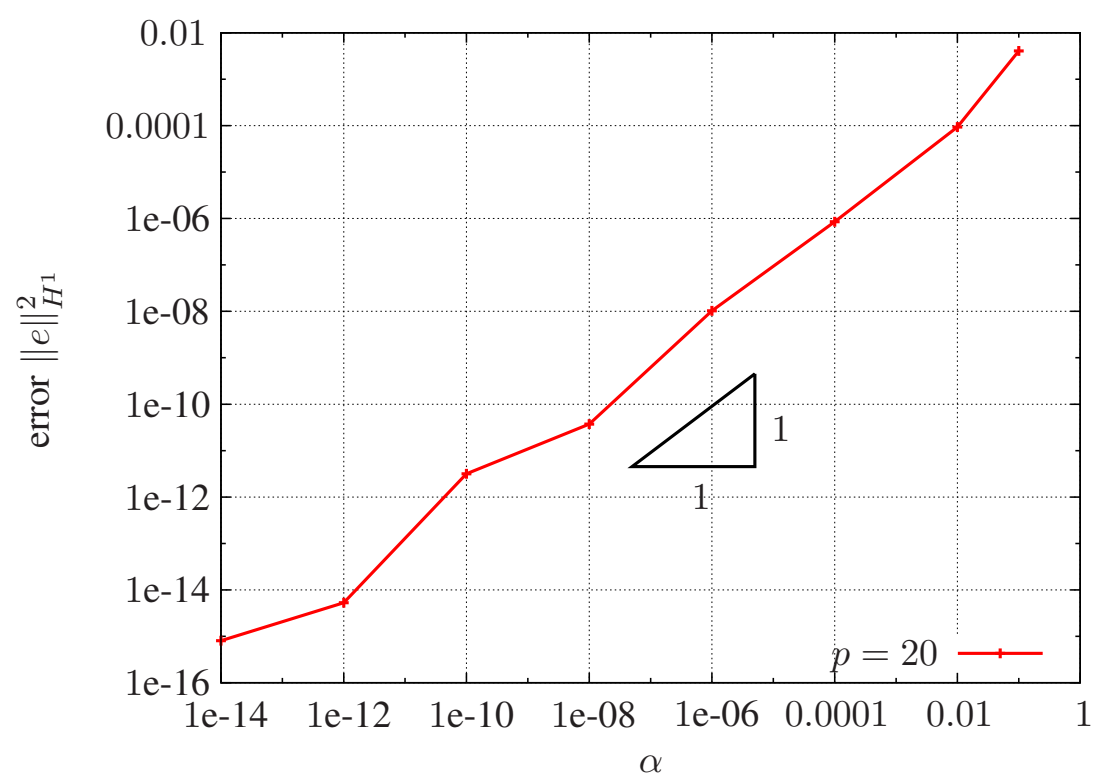

Figure 8: Influence of $\alpha$ on the error 


\subsubsection{Non-matching grid with three cells - case II}

In this example again three cells are used to mesh the fictitious domain resulting in a non-matching grid. However, this time the coordinates $X_{c}$ of the cells $\{0,0.8,1.2,2\}$ are chosen such that the middle element lies completely inside the hole. The results of the FCM computation with $p=20$ and $\alpha=10^{-14}$ are plotted together with the exact solution in Figure 9. The convergence in terms of the error $\|e\|_{H^{1}}^{2}$

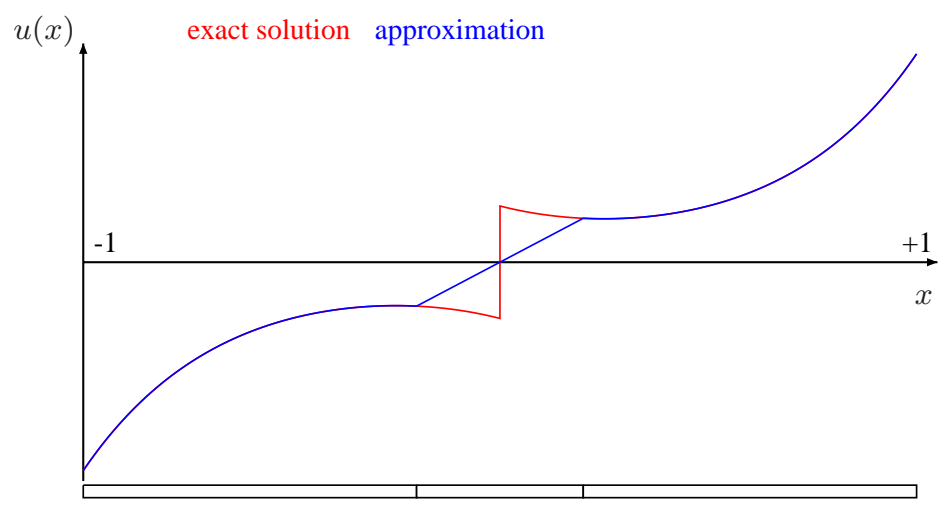

Figure 9: Comparison of exact solution and FCM approximation with $p=20$ and $\alpha=10^{-14}$

of a $p$-extension applying the FCM for different values of $\alpha$ is plotted in Figure 10. First of all, we observe a similar behaviour as in the previous example, i.e. an exponential convergence can be obtained which is limited by the value of $\alpha$. However, increasing the polynomial degree further on can result also in an increase of the error. This effect can be explained by the poor conditioning of the resulting equation system observed by the increase of the number of iterations of the preconditioned conjugate gradient method which is applied to solve the overall equation system. The poor conditioning is due to the fact that one cell is completely inside the hole and therefore almost no stiffness is related to the corresponding degrees of freedom of that element. Increasing the polynomial degree further on deteriorates the situation and therefore round-off error start to accumulate. Considering the scale of the $y$-axis of Figure 10 reveals that still very accurate results can be obtained with the FCM. Figure 11 presents, as in the previous examples, the dependency of the error $\|e\|_{H^{1}}^{2}$ on $\alpha$. Although the conditioning problem interferes this investigation, the numerical results are again in good coherence with Theorem 7 , stating that the error in quadratic energy converges linearly in $\alpha$. 


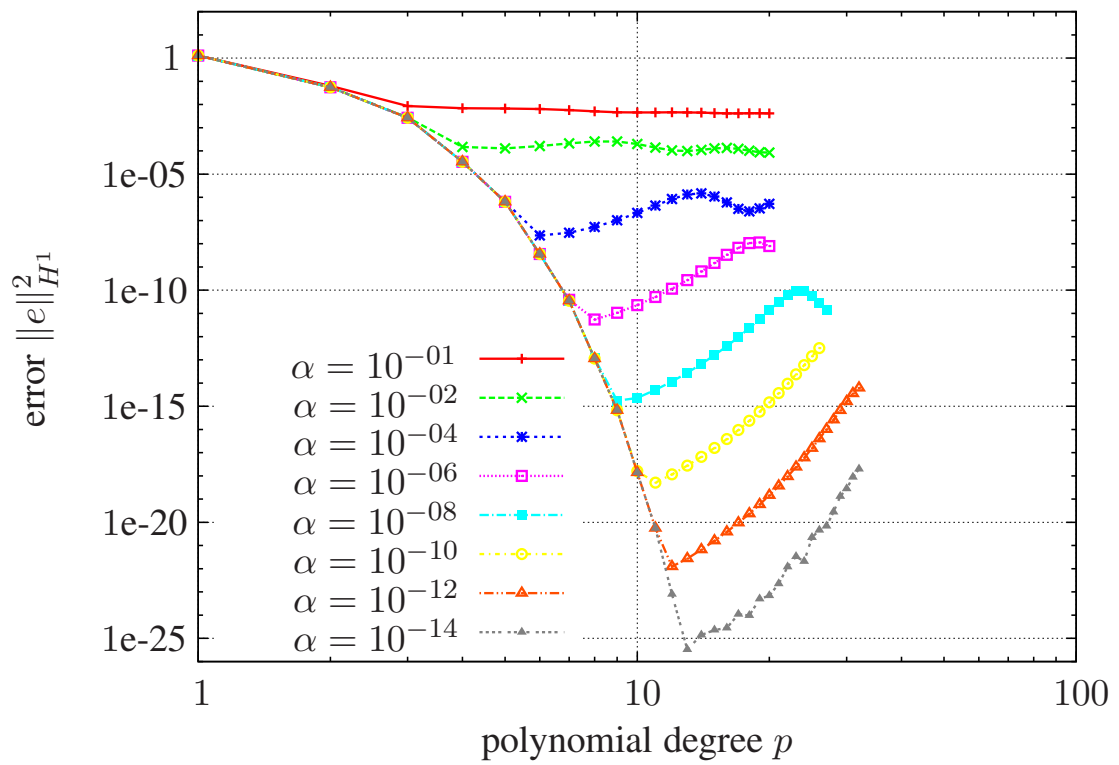

Figure 10: $p$-Extension on mesh with three elements for different $\alpha$ values

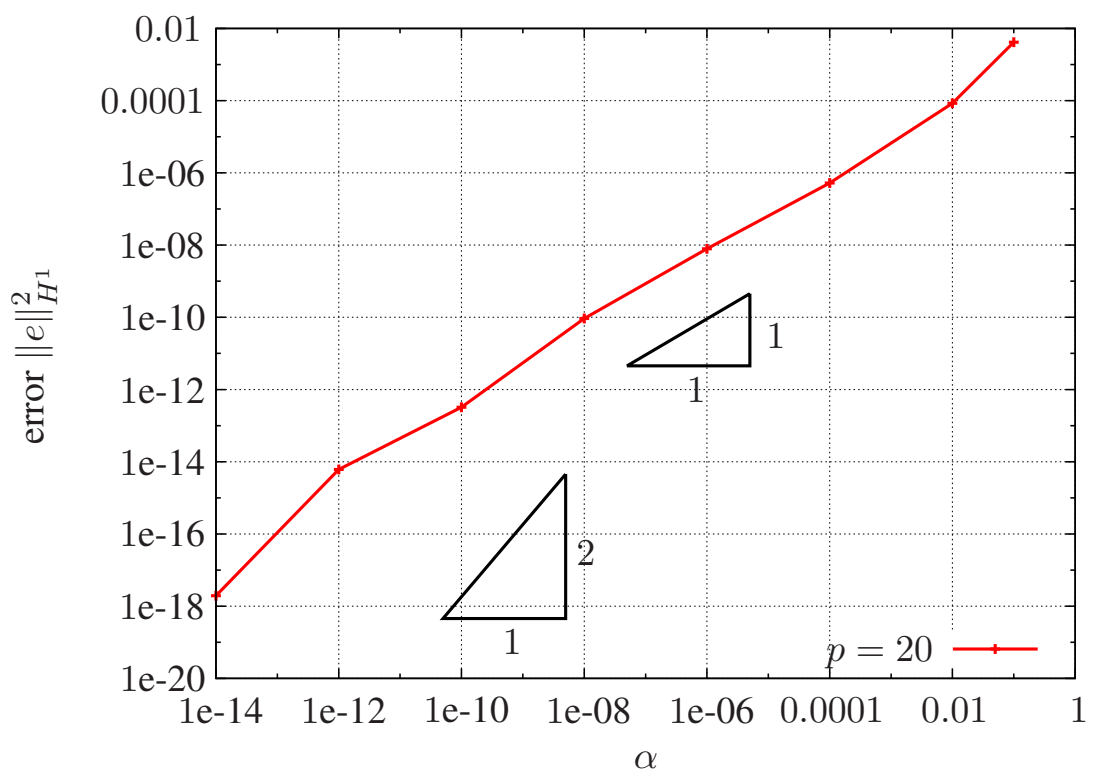

Figure 11: Influence of $\alpha$ on the error 


\subsubsection{Influence of integration error}

Next, we consider computations based on a mesh with two equidistant cells, i.e. with nodal coordinates $X_{c}$ corresponding to $\{0,1,2\}$. A comparison of the exact solution with the FCM approximation with $p=20$ and $\alpha=10^{-14}$ is given in Figure 12. Note, that an exact integration has been carried out by applying a composed Gaussian integration. Since the convergence of the error $\|e\|_{H^{1}}^{2}$ with respect to

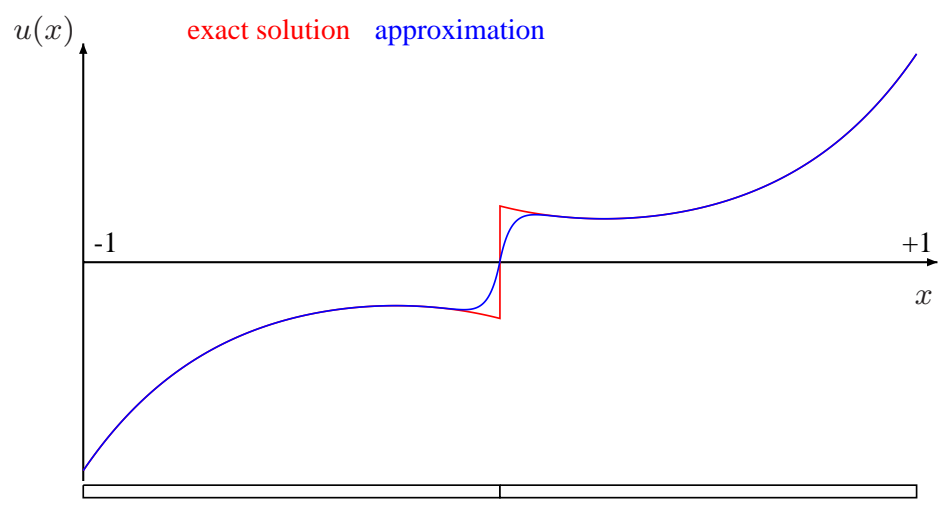

Figure 12: Comparison of exact solution and FCM approximation with $p=20$ and $\alpha=10^{-14}$

a $p$-extension and the choice of $\alpha$ is very similar to the results of the non-matching grid with the hole being completely located inside the middle element, see Section 5.4.3, they are not presented here in detail. Summarizing the results, a $p$-extension on the (non-matching) mesh with two cells yields an exponential convergence up to the error $\alpha$ in quadratic energy $\|e\|_{H^{1}}^{2}$.

Since in two and three spatial dimensions an exact integration of the stiffness and mass matrix is in general impossible, we investigate also the influence of the quality of the quadrature. The integration of the matrices of the two equidistant cells can be carried out exactly when applying $n_{s c}=\frac{1-0}{1-0.75}=4$ sub-cells with $n_{G}=p+1$ Gaussian points on each sub-cell. Here we choose $n_{s c}$ such that we can not perform an exact integration in order to investigate the influence of the quadrature. We choose $\alpha=0$ in order exclude a modelling error and focus only on the influence on the integration. In Figure 13 the convergence of the error $\|e\|_{H^{1}}^{2}$ is plotted as a function of the number of sub-cells $n_{s c}$ in a double logarithmic style. From the figure it is evident that the convergence is up to $n_{s c}^{-2}$ in quadratic energy. 


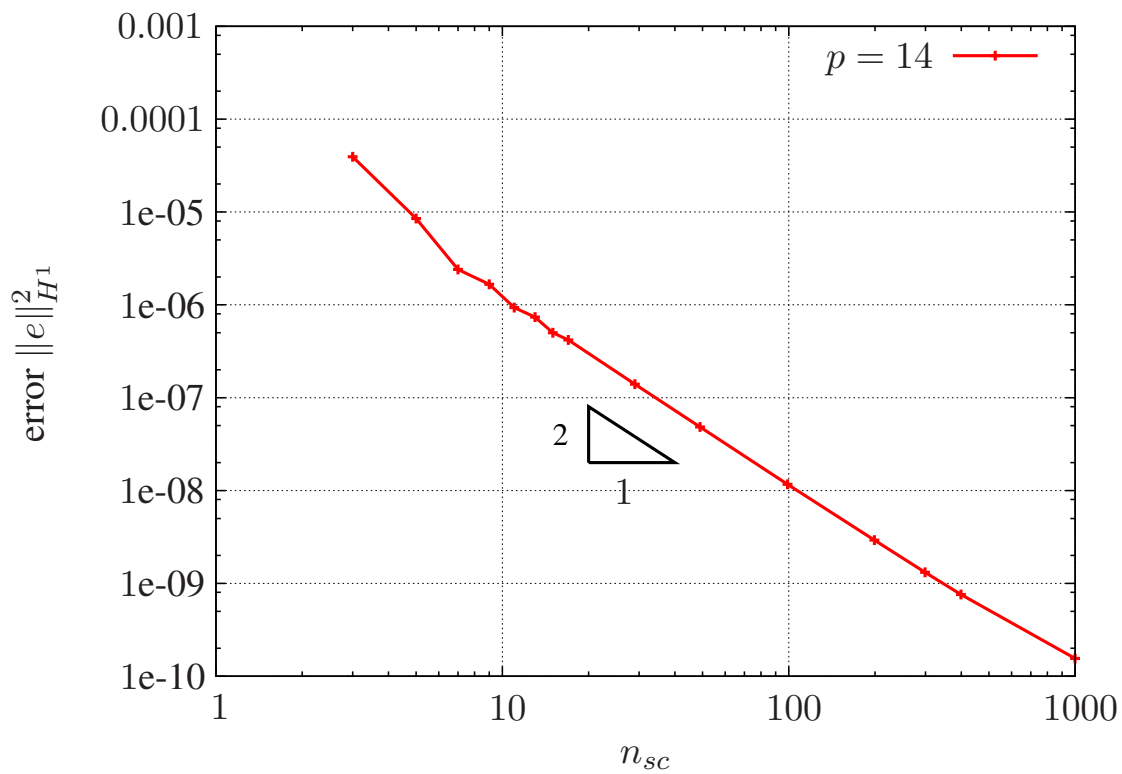

Figure 13: Convergence with respect to the number of sub-cells $n_{s c}$ each of with $n_{G}=p+1$ Gaussian points 


\section{A 2D benchmark of linear elasticity}

Finally, we study a two-dimensional benchmark problem of linear elasticity which was defined in [24] to compare different adaptive finite element strategies. The benchmark problem is a two-dimensional plate under plane strain condition. Due to symmetry it is sufficient to discretize one quarter of the system, see Figure 14. The width and height are $b=h=100 \mathrm{~mm}$ and the radius is $r=10 \mathrm{~mm}$. Linear isotropic elasticity with Young's modulus $E=206900 \mathrm{MPa}$ and Poisson's ratio of $\nu=0.29$ is assumed. The plate is loaded by a traction of $p=450 \mathrm{MPa}$. The quantities to be computed are given in Table 1 in which also the reference values are listed. The plate is discretized with $2 \times 2$ quadrilateral

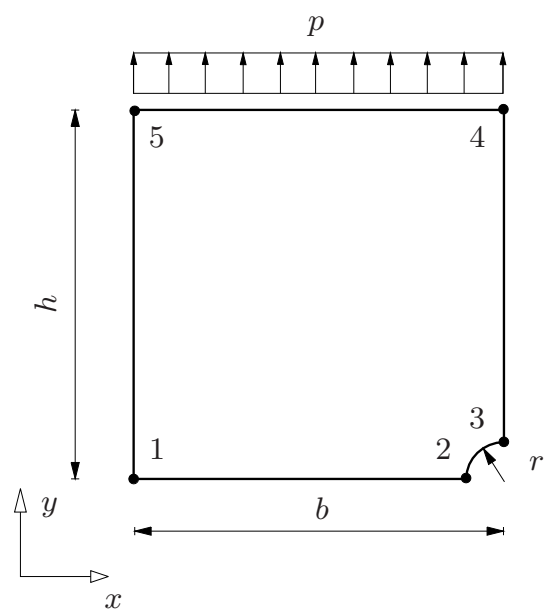

Figure 14: Square plate with a circular hole taking advantage of symmetry

\begin{tabular}{|c|c|c|c|c|}
\hline \hline strain energy & \multicolumn{2}{|c|}{ node 2 } & node 4 & node 5 \\
$\mathcal{U}_{\text {ref }}[\mathrm{Nmm}]$ & $u_{x}[\mathrm{~mm}]$ & $\sigma_{y y}[\mathrm{MPa}]$ & $u_{y}[\mathrm{~mm}]$ & $u_{x}[\mathrm{~mm}]$ \\
\hline 4590.773146 & 0.021290 & 1388.732343 & 0.209514 & 0.076758 \\
\hline \hline
\end{tabular}

Table 1: Reference values

cells on which the tensor product space utilizing hierarchic shape functions $[25,4]$ is used to discretize the trial and test functions. In Figure 15 the FCM grid as well as the sub-cells that are introduced for integration purposes only are presented. The sub-cells are generated in a fully automatic way by means of a space partitioning scheme based on a quadtree. We set $\alpha=10^{-14}$ inside the hole and the quadtree is refined towards the boundary of the circle. The leafs of the quadtree correspond to the sub-cells that are used for the adaptive quadrature [2]. On each of the sub-cells a Gaussian quadrature is performed to accurately compute the stiffness matrix of the cell that is cut by the circle. As a first result the relative error in energy norm

$$
e_{\text {rel }}=\sqrt{\frac{\left|\mathcal{U}_{r e f}-\mathcal{U}\right|}{\mathcal{U}_{\text {ref }}}} 100[\%]
$$

is plotted in Figure 16 demonstrating clearly the exponential convergence of the $p$-extension. Similar to the one-dimensional test example the optimal type of convergence for the FCM performing a $p$ extension can be obtained also in the case of holes in two-dimensions. In order not to hinder the optimal 

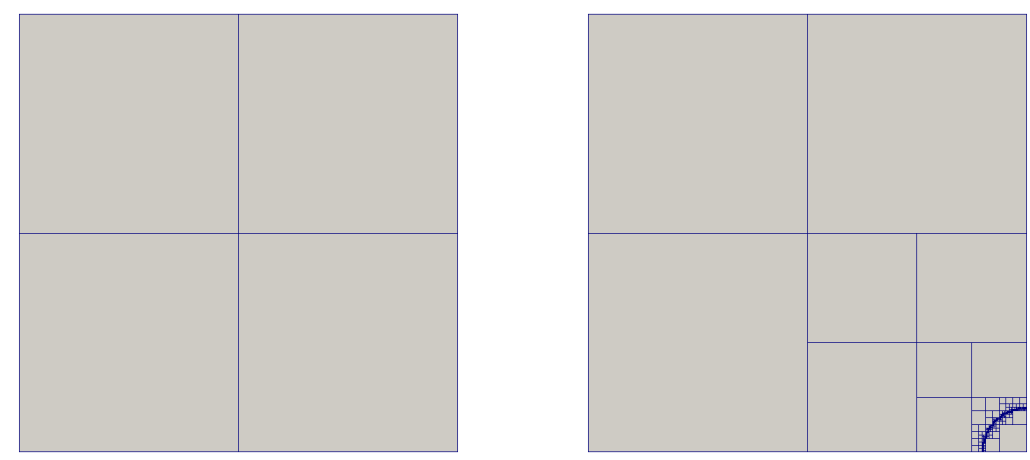

Figure 15: FCM grid with $2 \times 2$ cells (left) and corresponding sub-cells for integration purposes (right)

convergence rate, the integration of the cell cut by the circle has been carried out with a very high accuracy, demonstrating that the exponential convergence can be observed within a $p$-extension from $p=1$ up to $p=20$. In practice, however, a finer grid of cells with a moderate polynomial degree of $p=6, \ldots, 8$ would lead to accuracies which are relevant for engineering decisions. The FCM results for

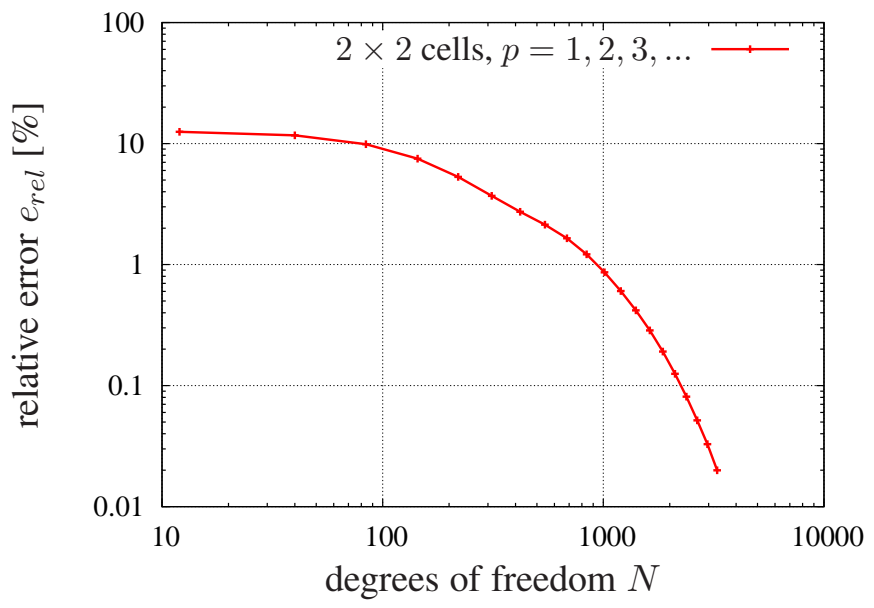

Figure 16: Convergence of the error in energy norm for a $p$-extension on $2 \times 2$ cells

the displacement $u_{y}$ at point 4 and the displacement $u_{x}$ at point 5 are depicted in Figure 17 showing a fast convergence of the $p$-extension also for point-wise quantities like displacements. More challenging than the results at points 4 and 5 are those quantities which are computed directly at the boundary of the hole. Therefore we study also the convergence of the displacement $u_{x}$ and stress component $\sigma_{y y}$ at point 2, see Figure 18. Again, a fast convergence towards the reference values can be observed even for the stress component $\sigma_{y y}$. 

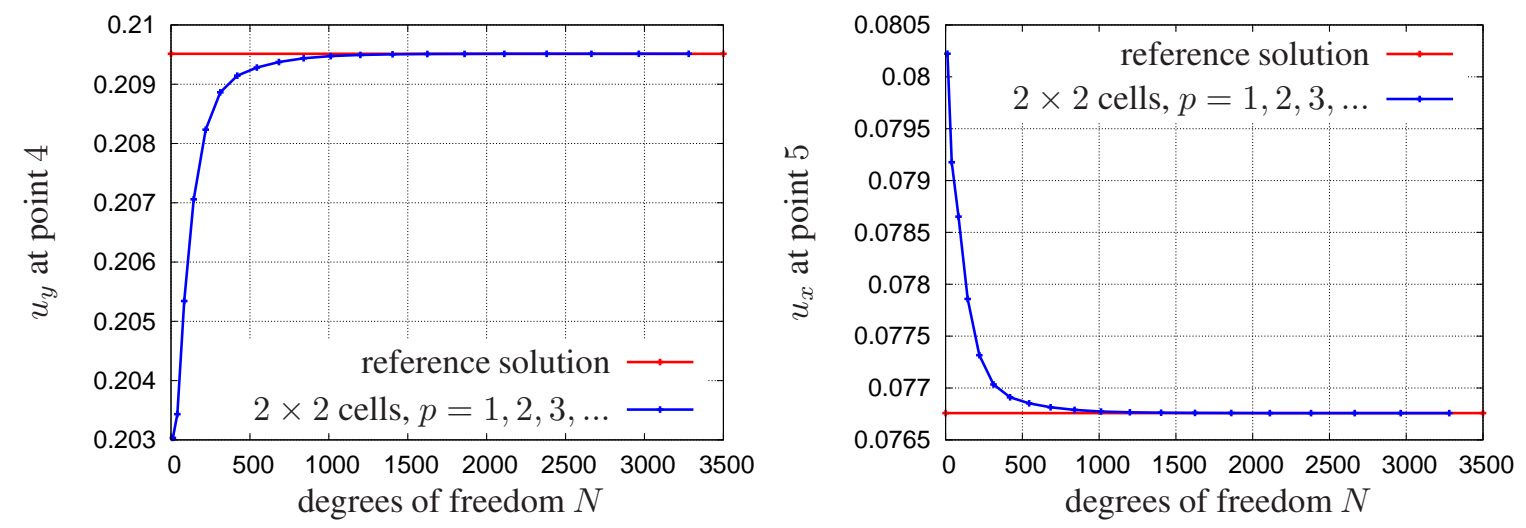

Figure 17: Convergence of the displacement $u_{y}$ at point 4 and $u_{x}$ at point 5 for a $p$-extension on $2 \times 2$ cells
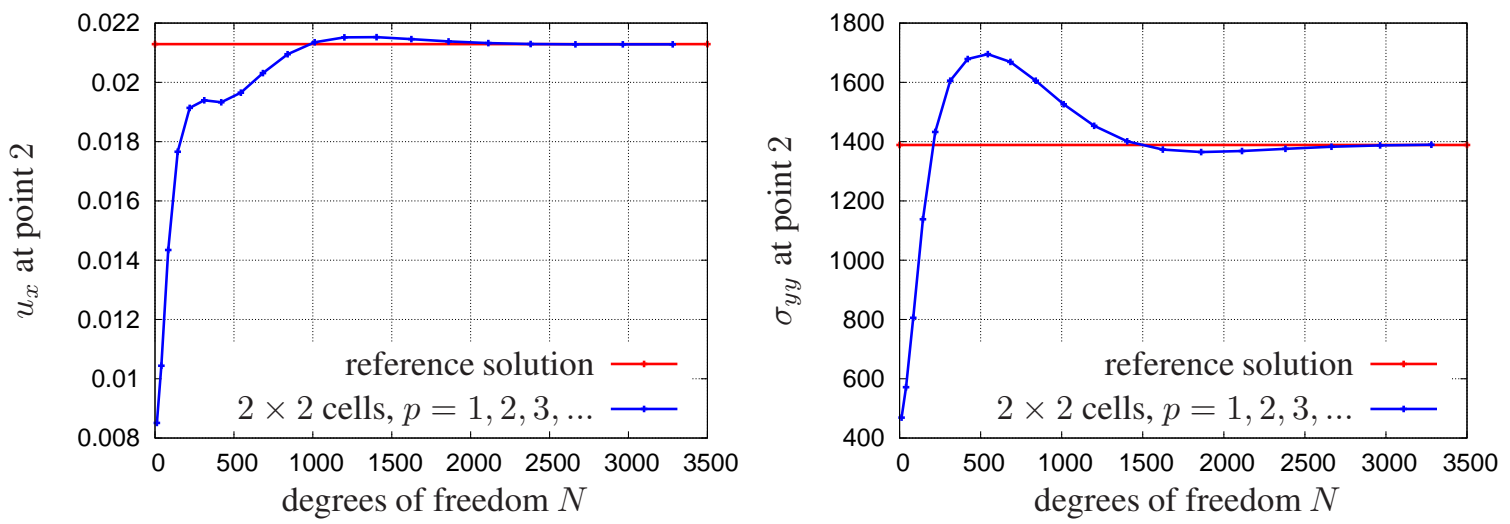

Figure 18: Convergence of the displacement $u_{x}$ and stress component $\sigma_{y y}$ at point 2 for a $p$-extension on $2 \times 2$ cells 


\section{Conclusions}

A mathematical analysis of the finite cell method has been presented, proving exponential rate of convergence which has been observed earlier by numerical investigations, only. Necessary smoothness conditions of the exact solution are similar to those which have to be assumed for the classical $p$ version of the finite element method. Furthermore, the dependence of an inherent modelling error on the computational scheme's penalisation parameter was proved and numerically confirmed. This high order fictitious domain approach thus not only yields significant advantages over FEM concerning engineering applications by virtually relieving from the necessity to generate a finite element mesh, it also guarantees convergence properties, which are inachievable for low order methods.

This paper concentrates on Neumann boundary conditions at the transition from the physical to the fictitious domain. Numerical experiments using Nitsche's method [12] to apply Dirichlet conditions show $[23,18]$ that also in this case exponential convergence rates can be obtained. A mathematical investigation confirming this observation still has to be done in future work.

\section{References}

[1] A. Abedian, J. Parvizian, A. Düster, H. Khademyzadeh, and E. Rank. The Finite Cell Method for Elasto-Plastic Problems. In Proceedings of the Tenth International Conference on Computational Structures Technology, Civil-Comp Press, 2010.

[2] A. Abedian, J. Parvizian, A. Düster, H. Khademyzadeh, and E. Rank. Performance of different integration schemes in facing discontinuites in the finite cell method. International Journal of Computational Methods, 10(3):1350002/1-24, 2013.

[3] A. Abedian, J. Parvizian, A. Düster, and E. Rank. The finite cell method for the $\mathrm{J}_{2}$ flow theory of plasticity. Finite Elements in Analysis and Design, 69:37-47, 2013.

[4] A. Düster, H. Bröker, and E. Rank. The p-version of the finite element method for threedimensional curved thin walled structures. International Journal for Numerical Methods in Engineering, 52:673-703, 2001.

[5] A. Düster, A. Niggl, and E. Rank. Applying the $h p-d$ version of the FEM to locally enhance dimensionally reduced models. Computer Methods in Applied Mechanics and Engineering, 196:35243533, 2007.

[6] A. Düster, J. Parvizian, and E. Rank. Topology optimization based on the finite cell method. Proceedings in Applied Mathematics and Mechanics, 10:151-152, 2010.

[7] A. Düster, J. Parvizian, Z. Yang, and E. Rank. The finite cell method for three-dimensional problems of solid mechanics. Computer Methods in Applied Mechanics and Engineering, 197:37683782, 2008.

[8] A. Düster and E. Rank. Die Finite Cell Methode - Eine Fictitious Domain Methode mit FinitenElement Ansätzen hoher Ordnung. In GAMM Rundbrief, volume 2, pages 6-13, 2011.

[9] A. Düster, H.-G. Sehlhorst, and E. Rank. Numerical homogenization of heterogeneous and cellular materials utilizing the finite cell method. Computational Mechanics, 50:413-431, 2012. 
[10] T. J. R. Hughes, J. A. Cottrell, and Y. Bazilevs. Isogeometric analysis: CAD, finite elements, NURBS, exact geometry and mesh refinement. Computer Methods in Applied Mechanics and Engineering, 194:4135-4195, 2005.

[11] M. Joulaian and A. Düster. Local enrichment of the finite cell method for problems with material interfaces. Computational Mechanics, DOI: 10.1007/s00466-013-0853-8, 2013.

[12] J. Nitsche. Über ein Variationsprinzip zur Lösung von Dirichlet-Problemen bei Verwendung von Teilräumen, die keinen Randbedingungen unterworfen sind. Abhandlungen aus dem Mathematischen Seminar der Universität Hamburg, 36(1):9-15, 1971.

[13] J. Parvizian, A. Düster, and E. Rank. Finite cell method - h- and p-extension for embedded domain problems in solid mechanics. Computational Mechanics, 41:121-133, 2007.

[14] J. Parvizian, A. Düster, and E. Rank. Topology optimization using the finite cell method. Optimization and Engineering, 13:57-78, 2012.

[15] E. Rank. Adaptive remeshing and h-p domain decomposition. Computer Methods in Applied Mechanics and Engineering, 101:299-313, 1992.

[16] E. Rank, S. Kollmannsberger, Ch. Sorger, and A. Düster. Shell Finite Cell Method: A High Order Fictitious Domain Approach for Thin-Walled Structures. Computer Methods in Applied Mechanics and Engineering, 200:3200-3209, 2011.

[17] E. Rank, M. Ruess, S. Kollmannsberger, D. Schillinger, and A. Düster. Geometric modeling, Isogeometric Analysis and the Finite Cell Method. Computer Methods in Applied Mechanics and Engineering, 249-252:104-115, 2012.

[18] M. Ruess, D. Schillinger, Y. Bazilevs, V. Varduhn, and E. Rank. Weakly Enforced Essential Boundary Conditions for NURBS-embedded and trimmed NURBS geometries on the basis of the Finite Cell Method. International Journal for Numerical Methods in Engineering, accepted for publication, 2013.

[19] V. K. Saul'ev. A method for automatization of the solution of boundary value problems on high performance computers. Dokl. Akad. Nauk SSSR 144 (1962), 497-500 (in Russian). English translation in Soviet Math. Dokl., 3:763-766, 1963.

[20] V. K. Saul'ev. On solution of some boundary value problems on high performance computers by fictitious domain method. Siberian Mathematical Journal, 4:912-925, 1963.

[21] D. Schillinger, A. Düster, and E. Rank. The $h p$ - $d$-adaptive finite cell method for geometrically nonlinear problems of solid mechanics. International Journal for Numerical Methods in Engineering, 89:1171-1202, 2012.

[22] D. Schillinger and E. Rank. An unfitted $h p$ adaptive finite element method based on hierarchical B-splines for interface problems of complex geometry. Computer Methods in Applied Mechanics and Engineering, 200:3358-3380, 2011.

[23] D. Schillinger, M. Ruess, N. Zander, Y. Bazilevs, A. Düster, and E. Rank. Small and large deformation analysis with the $\mathrm{p}$ - and $\mathrm{B}$-spline versions of the finite cell method. Computational Mechanics, 50:445-478, 2012. 
[24] E. Stein, editor. Error-Controlled Adaptive Finite Elements in Solid Mechanics. John Wiley \& Sons, 2002.

[25] B.A. Szabó and I. Babuška. Finite element analysis. John Wiley \& Sons, 1991.

[26] Z. Yang, S. Kollmannsberger, A. Düster, M. Ruess, E. Garcia, R. Burgkart, and E. Rank. Nonstandard bone simulation: interactive numerical analysis by computational steering. Computing and Visualization in Science, 14:207-216, 2012.

[27] Z. Yang, M. Ruess, S. Kollmannsberger, A. Düster, and E. Rank. An efficient integration technique for the voxel-based finite cell method. International Journal for Numerical Methods in Engineering, 91:457-471, 2012. 